\title{
Pronounced morphological changes in a southern active zone on comet 67P/Churyumov-Gerasimenko
}

\author{
P. H. Hasselmann ${ }^{1}$, M. A. Barucci ${ }^{1}$, S. Fornasier ${ }^{1}$, D. Bockelée-Morvan ${ }^{1}$, J. D. P. Deshapriya ${ }^{1}$, C. Feller ${ }^{1}$, \\ J. Sunshine ${ }^{2}$, V. Hoang ${ }^{1,3}$, H. Sierks ${ }^{4}$, G. Naletto ${ }^{5,6,7}$, P. L. Lamy ${ }^{8}$, R. Rodrigo ${ }^{9,10}$, D. Koschny ${ }^{11}$, B. Davidsson ${ }^{12}$, \\ J.-L. Bertaux ${ }^{8}$, I. Bertini ${ }^{5}$, D. Bodewits ${ }^{13}$, G. Cremonese ${ }^{14}$, V. Da Deppo ${ }^{7}$, S. Debei ${ }^{15}$, M. Fulle ${ }^{17}$, P. J. Gutierrez ${ }^{18}$, \\ C. Güttler ${ }^{4}$, J. Deller ${ }^{4}$, W.-H. Ip ${ }^{19,20}$, H. U. Keller ${ }^{21,22}$, L. M. Lara' ${ }^{18}$, M. De Cecco ${ }^{16}$, M. Lazzarin ${ }^{13}$, \\ J. J. L. López-Moreno ${ }^{18}$, F. Marzari ${ }^{5}, \mathrm{X} . \mathrm{Shi}^{4}$, and C. Tubiana ${ }^{4}$ \\ (Affiliations can be found after the references)
}

Received 24 July 2018 / Accepted 23 January 2019

\begin{abstract}
A smooth deposit in the southern Khonsu region has been seen in ESA/Rosetta observations as active during the second half of 2015 , when the southern summer coincided with the perihelion passage of 67P/Churyumov-Gerasimenko (67P). Image color sequences acquired by the OSIRIS instrument in the period of January 2015 to July 2016, pre- and post-perihelion, show the occurrence of several small transient events as well as three massive outbursts ( $\sim 10$ to 1500 tons). High spatial resolution images taken one year and a half apart allowed us to track a variety of sources: the formation of cavities that are $1.3-14 \mathrm{~m}$ deep, ice-enriched patches, scarp retraction, and a second $50 \mathrm{~m}$-wide boulder. We then estimated their masses and the dust mass of their corresponding plumes and outbursts. In particular, the deformation left by that boulder and its lack of talus may provide evidence for the lifting and subsequent falling back to the surface of large blocks. We calculate that a minimum vapor production rate of $1.4 \times 10^{24} \mathrm{~m}^{-2} \mathrm{~s}^{-1}$ is required to lift such an object. The comparison of the masses that are lost in the new cavities to the dust mass of outbursts gives indirect evidence of highly volatile ice pockets underneath. The spectrophotometric analysis and boulder counting also provides evidence for cavities that formed only $30 \mathrm{~m}$ apart with different spectral slopes, two long-standing ice patches, and local variations in the boulder-size frequency distribution. All this points to sub-surface ice pockets with different degrees of depth. Finally, the total mass of the morphological changes compared to most recent calculations of the total released mass by activity on $67 \mathrm{P}$ is estimated to be between 1.5 and $4.2 \%$. This means that as many as about 25 similar active zones across the nucleus would be enough to sustain the entire cometary activity.
\end{abstract}

Key words. comets: individual: 67P/Churyumov-Gerasimenko - methods: miscellaneous

\section{Introduction}

The southern hemisphere of the nucleus of comet $67 \mathrm{P} /$ Churyumov-Gerasimenko (67P) experienced several morphological changes (El-Maarry et al. 2017) and spectral bluing (Fornasier et al. 2016) on the surface during perihelion approach (August 13, 2015, $r_{h}=1.243 \mathrm{AU}$ ) in the second half of 2015. Because of the obliquity of the rotation axis of the nucleus $\left(52^{\circ}\right.$, Mottola et al. 2014) the summer season in the southern hemisphere matches the perihelion approach. Accordingly, a rise in insolation, gas production (Keller et al. 2015), and cometary activity (Vincent et al. 2016a; Fornasier et al. 2019) is observed in some southern regions.

One of these regions, named Khonsu and physiographically defined by El-Maarry et al. (2016), has previously attracted attention because several exposed ice phenomena were observed, such as bright spots, blue patches, and spectral bluing over smoother terrains (Deshapriya et al. 2016). In addition to these, outbursts and morphological changes have also been noted, such as a 50-m-wide "jumping boulder", which was displaced downhill by an unnoticed activity during perihelion passage (El-Maarry et al. 2017). In particular, Vincent et al. (2016b) noted that two bright outbursts (labeled events 7 and 33 therein) originated from a very constrained zone in Khonsu (see Fig. 1 for its physiographic definition), a smooth deposit of $100 \times 700 \mathrm{~m}^{2}$ bounded by cliffs and the iconic pancake feature (El-Maarry et al. 2016). Deshapriya et al. (2018) roughly correlated event 7 of August 1, 2015 , to the vanishing of a high mass and the following collapse of two 20-m boulders lying on top. More recently, Laeuter et al. (2019) used the ROSINA ${ }^{1}$ instrument to detect the surface source of $\mathrm{H}_{2} \mathrm{O}, \mathrm{CO}_{2}, \mathrm{CO}$, and $\mathrm{O}_{2}$ gas emissions. Laeuter et al. (2019) located the Khonsu area near the Apis plateau as one of the main contributors, that is, over the same area as where the deposit is found.

All this establishes the deposit in Khonsu as one of the most active zones of the nucleus. The so-called Khonsu bank was therefore selected as one of the targets for the joint observation campaign by the ALICE $^{2}$, OSIRIS $^{3}$, MIRO $^{4}$, and VIRTIS instruments that was performed at low altitude $(8-20 \mathrm{~km})$ in the last months of the ESA/Rosetta mission. This campaign provided us with images at a decimeter spatial resolution. Therefore, we devote this study to a detailed description and quantification

\footnotetext{
Rosetta Orbiter Spectrometer for Ion and Neutral Analysis.

2 Ultraviolet Imaging Spectrometer.

Optical, Spectroscopic and Infrared Remote Imaging System.

4 Microwave Instrument for the Rosetta Orbiter.

Visible and Infrared Thermal Imaging Spectrometer.
} 




Fig. 1. Southern-equatorial Khonsu region and the bank. Rough boundaries of the Khonsu region as delimited by El-Maarry et al. (2016). Background composed of a false-color RGB figure from the NAC F41F23-F23 filter of the sequence obtained on May 2, 2015, UTC 15:09. The full extent of the region is roughly located between $0^{\circ}$ to $-62^{\circ}$ latitude. Khonsu is bordered by the Apis plateau in the north, the Atum outcrop in the east, Imhotep in the west, and Bes-Sobek in the south. Khonsu bank (highlighted) is naturally divided into two sections through an interruption of a cliff situated around $-20^{\circ}$ latitude. The High bank lies on the equatorial reach between $-10^{\circ}$ and $-20^{\circ}$ latitude, while the Low bank is located between $-20^{\circ}$ and $-30^{\circ}$ latitude in the southern outskirts, near the pancake feature.

of activity, activity sources, and the morphological and spectrophotometric changes that occurred in the Khonsu bank using multi-filter high-resolution images obtained by the OSIRIS cameras. Our goal is to understand the mass loss taking place in active zones of $67 \mathrm{P}$ nucleus. We were able to estimate volumes and masses and constrain the amount of total ejected mass.

\section{Observations and ancillary data}

OSIRIS/NAC observations. We rely on the multi-filter observations acquired by the Narrow-Angle Camera (NAC) in the OSIRIS (Keller et al. 2007). The NAC was developed for spectromorphological mapping and monitoring of transient events. It had a narrow field of view of $2.35^{\circ} \times 2.35^{\circ}$ and an angular resolution of $18.6 \mu \mathrm{rad} \mathrm{pixel}^{-1}$. The color sequences were acquired in a partial combination of 12 broad- and narrow-band filters ranging in the wavelength domain of 250-1000 nm. Each image was corrected for optical distortions, was radiometrically calibrated, and stored in 32-bit float data and converted into radiance factor ( $r_{F}$ or RADF, dimensionless) units (Tubiana et al. 2015).

For the morphological analysis, image color sequences framing the Khonsu bank were selected according to the epochs considered (pre-, near-, and post-perihelion) based on the best spatial resolution and/or illumination conditions. Therefore, we include images obtained during the target of opportunity (ToO) campaign, which were acquired in 2016 with the highest spatial resolution.

Concerning the associated activity emanating from Khonsu Bank, we focus on dates of reported events in the literature. During the same summer, between August 1, 2015, and September 30, 2015, the average time interval among the OSIRIS observation decreased to a step of $12 \mathrm{~min}$. Vincent et al. (2016b) undertook a major survey on cometary events, and discussed the completeness of the sample at great length. They stated that it is very unlikely that any bright outburst was missed during that period. In the same time period, the distance of Rosetta to the comet was $>300 \mathrm{~km}$, to only reapproach $<200 \mathrm{~km}$ in the beginning of November 2015. Very short and faint localized events therefore became unnoticeable after August 8, 2015. Furthermore, due to the high phase angle in which the observations were obtained, the conditions have favored the detection of night-side activity. Fornasier et al. (2019) have recently been able to detect 215 small emissions with the OSIRIS cameras, however. Most of the activity analyzed here was cataloged thanks to this study.

During the time-frame of event 7 in Vincent et al. (2016b), which was also cited in the introduction, Fornasier et al. (2019) subsequently observed a complex sequence of emissions occurring during spectrorotational sequences on August 1, 2015, which deserved closer inspection. Event 33 (also from Vincent et al. 2016b) on September 14, 2015, 18:47, on the other hand, was studied in its VIS colors, temperature, composition, mass and other properties using VIRTIS-M-VIS (Rinaldi et al. 2018) and VIRTIS-H (Bockelée-Morvan et al. 2017). Finally, we also report a previously undetected outburst emanating from Khonsu bank on December 13, 2015, $\left(r_{h}=1.87 \mathrm{AU}\right)$ in the waning period of activity.

Selected data are reported in Table 1. Information on filters, solar distance $\left(r_{h}\right), \mathrm{S} / \mathrm{C}$ distance $(\Delta)$, spatial resolution $\left(\mathrm{cm} \mathrm{pixel}^{-1}\right)$, phase angle $(\alpha)$, and sub-solar latitude (lat $\left.{ }_{\odot}\right)$ and longitude $\left(\operatorname{lon}_{\odot}\right)$ is specified for every included NAC color sequence.

Cartographic referencing. We produced ancillary images in the default NAC F22 $(649 \mathrm{~nm})$ frame using the OASIS image simulator (Jorda et al. 2010) supplied with the NAIF SPICE kernels (Acton 1996; Acton et al. 2018) released in November 2017 and the stereo-photogrammetric (SPG) SHAP7 model resampled to four million facets (Preusker et al. 2017). The ancillary images contain values of incidence angle $(i)$, emergence angle $(e)$, phase angle $(\alpha), \mathrm{S} / \mathrm{C}$ distance $(\Delta)$, central facet, and Cartesian coordinates per pixel. From the Cartesian coordinates, latitude and longitude were computed with respect to the Cheops reference frame (Preusker et al. 2015).

Image registration. Images from color sequences were obtained consecutively in offsets of tens of seconds in between acquisitions, thus inducing slight distortions in projection from one image to another. To coregister all images to a common frame, we ran an automatic feature detector (Rublee et al. 2011) that led to the homograph transformation of every image to the new frame and conserved the pixel-energy 
P. H. Hasselmann et al.: Pronounced morphological changes in a southern active zone on comet 67P/Churyumov-Gerasimenko

Table 1. List of included NAC color sequences.

\begin{tabular}{|c|c|c|c|c|c|c|c|}
\hline Surface sequences (UTC) & Filters $^{(a)}$ & $r_{h}(\mathrm{AU})$ & $\Delta(\mathrm{km})$ & $\mathrm{cm} \mathrm{pixel}^{-1}$ & $\alpha\left(^{\circ}\right)$ & lat $_{\odot}\left({ }^{\circ}\right)$ & $\operatorname{lon}_{\odot}\left({ }^{\circ}\right)$ \\
\hline NAC 2015-01-16, 01:27 & F22-F24 & 2.53 & 26.5 & 50 & 97.5 & 29.1 & 178.9 \\
\hline NAC 2015-05-02, 15:09 & $\begin{array}{l}\text { F23-F22-F41-F24-F71- } \\
\text { F51-F61-F28-F16-F15 }\end{array}$ & 1.73 & 123.2 & 230 & 60.8 & 3.0 & 226.2 \\
\hline NAC 2015-08-01, 08:00 & $\begin{array}{c}\text { F22-F23-F24-F27- } \\
\text { F28-F16-F15-F41-F51- } \\
\text { F61-F71 }\end{array}$ & 1.25 & 214.2 & 385 & 90.5 & -42.7 & 216.7 \\
\hline NAC 2016-01-28, 01:48 & $\begin{array}{l}\text { F22-F23-F24-F27- } \\
\text { F28-F41-F71-F61-F16 }\end{array}$ & 2.23 & 66.8 & 120 & 62.4 & -10.1 & 160.4 \\
\hline NAC 2016-06-25, 06:09 & F41-F22-F24-F23-F16 & 3.27 & 18.5 & 33 & 93.1 & 12.1 & 208.3 \\
\hline NAC 2016-06-25, 18:02 & F41-F22-F24-F23-F16 & 3.28 & 16.1 & 29 & 92.8 & 12.1 & 213.7 \\
\hline NAC 2016-07-02, 07:57 & F41-F22-F24-F23-F16 & 3.32 & 18.2 & 33 & 91.8 & 12.9 & 178.8 \\
\hline NAC 2016-07-16, 19:22 & $\begin{array}{c}\text { F22-F23-F24-F16-F27- } \\
\text { F41-F71 }\end{array}$ & 3.40 & 8.4 & 15.8 & 101.7 & 14.2 & 245.8 \\
\hline Activity sequences (UTC) & Filters $^{(a)}$ & $r_{h}(\mathrm{AU})$ & $\Delta(\mathrm{km})$ & $\mathrm{cm}_{\text {pixel }}{ }^{-1}$ & $\alpha\left(^{\circ}\right)$ & lat $_{\odot}\left({ }^{\circ}\right)$ & $\operatorname{lon}_{\odot}\left({ }^{\circ}\right)$ \\
\hline NAC 2015-08-01, 08:43 & $\begin{array}{c}\text { F22-F23-F24-F27- } \\
\text { F28-F16-F15-F41-F51- } \\
\text { F61-F71 }\end{array}$ & 1.25 & 214.2 & 385 & 90.5 & -42.8 & 60.7 \\
\hline NAC 2015-08-01, 10:51 & F22-F23-F24-F71 & " & 213.4 & 384 & 89.9 & $"$ & 358.5 \\
\hline NAC 2015-08-01, 11:21 & F22-F23-F24-F71 & " & 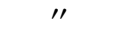 & '" & & $"$ & 343.9 \\
\hline NAC 2015-08-01, 11:51 & $\begin{array}{c}\text { F22-F23-F24-F27- } \\
\text { F28-F16-F15-F41-F51- } \\
\text { F61-F71 }\end{array}$ & " & 212.4 & 382 & " & " & 329.3 \\
\hline NAC 2015-08-01, 12:21 & $\prime \prime$ & " & 212.0 & " & $" 1$ & -42.9 & 314.7 \\
\hline NAC 2015-08-01, 20:55 & " & " & 207.7 & 374 & 89.7 & -43.0 & 65.4 \\
\hline NAC 2015-08-01, 21:25 & " & " & " & $\prime \prime$ & " & " & 50.9 \\
\hline NAC 2015-08-01, 21:55 & " & " & $" 1$ & " & $"$ & -43.1 & 36.3 \\
\hline NAC 2015-08-01, 22:25 & " & " & " & " & " & $\prime \prime$ & 21.7 \\
\hline NAC 2015-08-01, 22:55 & $" 1$ & " & 207.5 & " & $" 1$ & $"$ & 7.1 \\
\hline NAC 2015-08-01, 23:25 & " & " & " & " & $" 1$ & " & 352.6 \\
\hline NAC 2015-08-01, 23:55 & $\prime \prime$ & " & 206.9 & 372 & $"$ & " & 338.0 \\
\hline NAC 2015-09-10, 18:47 & $\mathrm{F} 22$ & & 314.1 & 565 & 99.6 & -51.5 & 160.5 \\
\hline NAC 2015-12-13, 01:13 & $\begin{array}{c}\text { F15-F24-F23-F51-F16- } \\
\text { F28-F27-F22-F71- } \\
\text { F61-F41 }\end{array}$ & 1.87 & 99.5 & 179 & 89.8 & -22.0 & 334.1 \\
\hline NAC 2015-12-13, 02:13 & " & " & " & " & " & " & 304.3 \\
\hline
\end{tabular}

Notes. The information is referenced to the center of the NAC F22 image contained in the sequence. ${ }^{(a)}$ Central wavelengths and bandwidth per filter: F22: orange, $649.2 \mathrm{~nm}(84.5 \mathrm{~nm})$; F23: green, $535.7 \mathrm{~nm}(62.4 \mathrm{~nm})$; F24: blue, $480.7 \mathrm{~nm}(74.9 \mathrm{~nm})$; F27: hydra, $701.2 \mathrm{~nm}(22.2 \mathrm{~nm})$; F28: red, $743.7 \mathrm{~nm}(64.1 \mathrm{~nm})$; F16: near-UV, $360 \mathrm{~nm}(51.1 \mathrm{~nm})$; F15: far-UV $269.3 \mathrm{~nm}(53.6 \mathrm{~nm})$; F41: near-IR, $882.1 \mathrm{~nm}(65.9 \mathrm{~nm})$; F51: ortho, 805.3 (40.5 nm); $\mathrm{F} 61: \mathrm{Fe}_{2} \mathrm{O}_{3}, 931.9 \mathrm{~nm}(34.9 \mathrm{~nm}) ; \mathrm{F} 71$ : IR, $989.3 \mathrm{~nm}(38.2 \mathrm{~nm})$.

(van der Walt, et al. 2014). The pixels of all images were therefore realigned to the standard NAC F22 frame. However, small artifacts may be present in shadow lines when the phase angle is sufficiently high.

In the next step, the color sequences are displayed in falsecolor RGB format generated using STIFF (Bertin 2012). RGB is a qualitative data visualization of variegations in three image channels. The filter combination used to generate the RGB composites can vary, therefore it is detailed in the figure captions.

Topographic-photometric correction. When topographicphotometric albedo corrections were required, we applied the Hapke isotropic multiple scattering approximation (IMSA model, see equation in Hapke 2008) with porosity-factor correction and disregarding shadow-hiding (SHOE) and coherentbackscattering (CBOE) opposition effects in the reference NAC F22 image. As all images studied here were obtained far from small phase angles, we ignored the opposition effect.
Hapke parameters reported in Fornasier et al. (2015) or Hasselmann et al. (2017) were used as default depending on whether images were taken pre- or post-perihelion. To improve the image-based correction, we minimized the singlescattering albedo $\left(w_{\lambda}\right)$ and average roughness slope $(\bar{\theta})$ using the L-BFGS-B algorithm (limited memory optimization $\operatorname{code}^{6}$; Zhu et al. 1997; Hasselmann et al. 2016, 2017). The topographicphotometric correction converts RADF into an approximated normal albedo $A_{0, \lambda=649}^{\prime} \mathrm{nm}$, a quantity invariant to incidence, emergence, and phase angles. This quantity is different from the nominal definition of a normal albedo, which also involves a correction for the opposition surge (Shkuratov et al. 2011).

Context of the Khonsu bank during Rosetta observations. In order to understand the conditions to which Khonsu bank was subject when Rosetta data were acquired, we performed

\footnotetext{
${ }^{6}$ https://docs.scipy.org/doc/scipy/reference/generated/ scipy.optimize.fmin_l_bfgs_b.html
} 


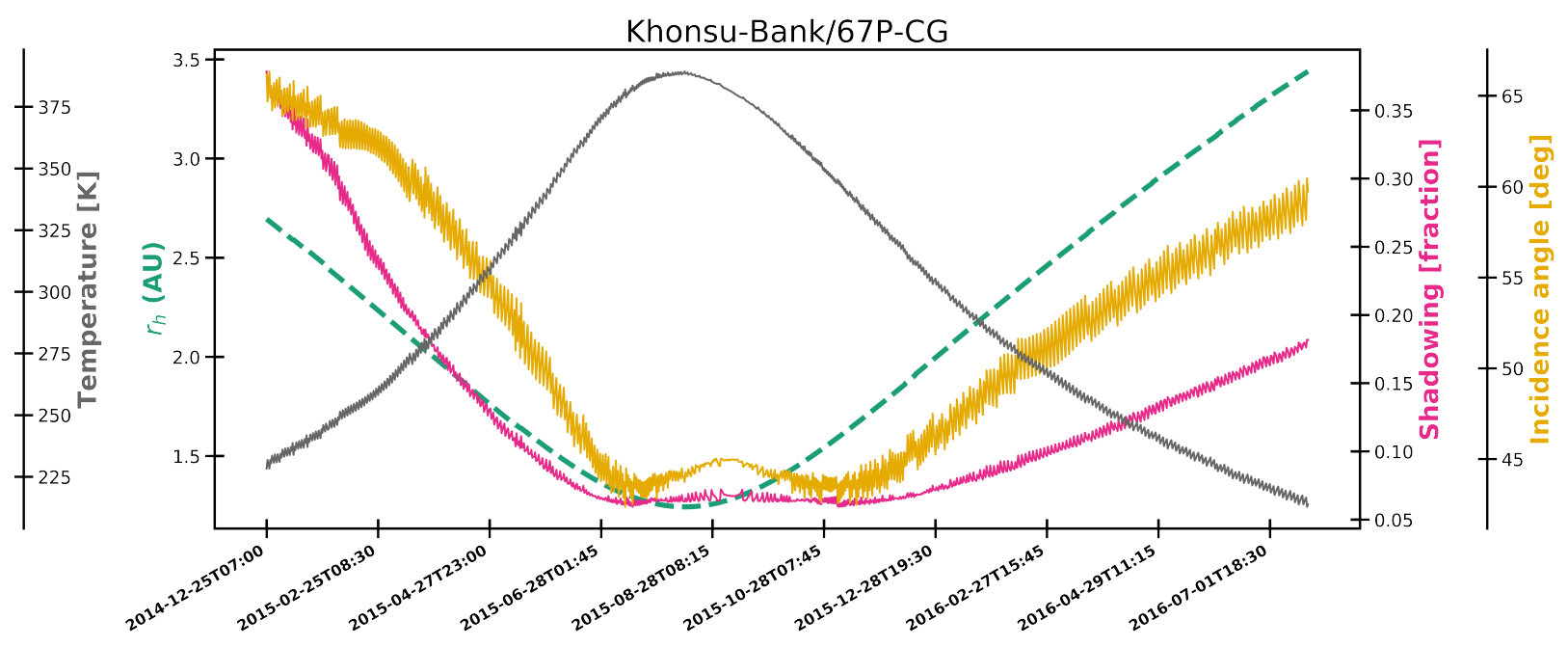

Fig. 2. Time-line of the Khonsu bank. Joint behavior of median solar distance $\left(r_{h}, \mathrm{AU}\right)$, median incidence angle (deg), median illuminated surface temperature (K), and median amount of shadow (\%) over DLR SPG SHAP7 168377 facets that describe the zone from December 12, 2014, to July 24,2016 . Every point was sampled on a cometary hour corresponding to 12 a.m at $\left(-22^{\circ}, 199^{\circ}\right)$.

the following exercise: we calculated the solar distance, incidence angle, bolometric irradiance and amount of shaded surface over SPG SHAP7 facets that describe the bank for every day at 12 a.m. (at $\left.-22^{\circ}, 199^{\circ}\right)$ in cometary hours from December 12 , 2014, to July 24, 2016. From the irradiance we estimated the local surface gray-body temperature over illuminated facets by considering the Stefan-Boltzmann law for an emissivity of 0.97 (Spohn et al. 2015; Keller et al. 2015). It is beyond the scope of this study to undertake complex thermal modeling.

In Fig. 2 we plot the joint median behavior of the calculated quantities through UTC time. During 2014, the positive sub-solar latitude $\left(\sim 32^{\circ}\right)$ left Khonsu bank with $\sim 40 \%$ of its surface predominantly in shadows due to oblique irradiation $\left(i \approx 65^{\circ}\right)$ at the maximum solar input. At this time, the zone was barely observable, and studies were mostly conducted in the northern hemisphere. Until the end of June 2015 (lat $_{\text {sun }} \sim-24^{\circ}$, $\left.r_{h} \sim 1.4 \mathrm{AU}\right)$, the temperature, shadowing, and incidence angle evolved monotonically. Khonsu became visible from an average $\mathrm{S} / \mathrm{C}$ distance of $120 \mathrm{~km}$.

The period of highest solar input begins in mid-July and ends in October 2015 (lat $\left._{\text {sun }} \sim-45^{\circ}, r_{h} \sim 1.3 \mathrm{AU}\right)$, during which the shaded surface dropped to $<10 \%$ and the surface temperature reached $\sim 380 \mathrm{~K}$. The Khonsu bank was highly active from the end of July until mid-December 2015 (see Sect. 6).

After mid-November 2015, the local solar incidence angle increased as the nucleus crossed beyond 1.8 AU. Shadowing and surface temperature again began to evolve monotonically, but at a different rate than at pre-perihelion condition. Mid-day shadowing did not exceed $20 \%$, meaning that Khonsu remained observable, which explains why the southern hemisphere was only mapped in detail after January 2016.

\section{Method}

\subsection{Measurement of the length}

Framing is regarded as a section of solid angle that encloses a scene observed by a camera. Considering that all optical geometric defects have been minimized in the OSIRIS images, we can therefore measure any length through the generalized distance between two points of position $(r, \theta, \lambda)$ and $\left(r^{\prime}, \theta^{\prime}, \lambda^{\prime}\right)$ in spherical coordinates with an origin with respect to the camera position:

$$
\begin{aligned}
& \left\|\mathbf{d}-\mathbf{d}^{\prime}\right\| \\
& =\sqrt{r^{2}+r^{\prime 2}-2 r^{\prime} r\left(\sin \theta \sin \theta^{\prime} \cos \left(\lambda-\lambda^{\prime}\right)+\cos \theta \cos \theta^{\prime}\right),}
\end{aligned}
$$

where $r$ and $r^{\prime}$ are the camera-to-surface distances of two given points given by the surface of the shape model, $\theta$ and $\theta^{\prime}$ are the $X$-axis image frame coordinates of the two points in radians, referenced to the image center, while $\lambda$ and $\lambda^{\prime}$ are the same, but for the $Y$-axis image frame coordinates. To obtain $\theta, \theta^{\prime}, \lambda$, and $\lambda^{\prime}$, we multiplied their numerical position in the image frame by the OSIRIS NAC angular resolution. Uncertainties were calculated through the standard deviation of several lengths measured for a given structure. For round structures, lengths were taken by precessing them every $\sim 10^{\circ}$ with respect to the apparent center.

\subsection{Shadow length}

The shadow cast by landmarks (e.g., craters, hills, and cliffs) is often relied on to estimate their depth or height in the imageresolved studies of bodies in the solar system (Arthur 1974; Chappelow \& Sharpton 2002; El-Maarry et al. 2017). As all the images of our dataset were acquired at large phase angles, this enables us to use the shadow casting length as a proxy of dimension. From the shadow length $L_{\text {shadow }}$ measured through the generalized distance between the top of the structure and the tip of its shadow, we applied the formula of shadow casting to obtain the height $h$ :

$h=L_{\text {shadow }} \times \tan (\pi / 2-i)$.

The incidence angle $i$ was otherwise estimated from the average of all facets intercepting the tops and tips of the shadow. To robustly estimate $h$, we manually traced the shadow profile every 5-10 pixels and regarded the projected Sun direction (azimuth angle) to the image frame. For small and round landmarks, we had only a few profiles near the summit. Finally, the uncertainties were calculated based on their standard deviations, which comprises manual errors as well as shape model uncertainties.

\subsection{Boulder-size frequency distribution}

The boulder-size frequency distribution is a geophysical analysis that gives access to fragmentation processes and to the 
cohesive strength of a certain surface region (Boulton 1978; Lee et al. 1996). The slope of their cumulative distribution allows us to assess the amount of grinding that the material was subjected to Golombek \& Rapp (1997). Generally, flat slopes represent materials that are resilient to further grinding, while steep slopes correlate with brittle materials. However, the distributions are only comparable if the fragmentation process can be considered the same or similar. For features that also exhibit spatially resolved regolith fields in their surroundings, we performed this analysis.

The method was applied as follows: we counted positive reliefs in the NAC F22 images that cast a shadow greater than 3 pixels, thus $45-100 \mathrm{~cm}$ at the spatial range of images obtained during the observational campaign. The length for barely visible reliefs was measured transversally to the shadow line defined by its shape. To compute their surface, we assumed them to be circumcircles (Pajola et al. 2015). The manual length error associated with this measurement was fixed to 2 pixels.

When the visual boulder counting was exhausted for a certain field, where "field" is qualitatively defined as a visual overdensity of positive reliefs, we calculated the logarithmic cumulative distribution normalized by the total surface enclosed by all counted reliefs as a logarithmic function of the boulder size in meters (Golombek \& Rapp 1997; Pajola et al. 2015).

To measure the distribution slope, we modeled the cumulative distribution with a power-law distribution. In the interval of data completeness, the logarithmic cumulative distribution is approximatively linear, which allows us to derive the slope by simple linear regression. Therefore, using orthogonal distance regression ${ }^{7}$ (Boggs \& Rogers 1990), we estimated their power-law index and associated error.

\subsection{Minimum lifting gas production rate}

The minimum $\mathrm{H}_{2} \mathrm{O}$ gas production rate required for the lifting is a question that it is worth addressing for some scattered boulders we detected in the Khonsu Bank. For lifting to take place, the gas acceleration must overcome the local gravity $g$. We refer to El-Maarry et al. (2017, Supp. Mat. S2) for the detailed mathematical formulation. The authors have applied the following formula to compute the minimum $\mathrm{H}_{2} \mathrm{O}$ gas production rate to lift a 30-m-wide boulder that was also located in Khonsu (jumping boulder 1 hereafter). The equation for any given boulder radius is

$Z_{\mathrm{gas}}>\frac{8 g \rho R_{\mathrm{eff}}}{3 C_{D} c_{g}}=5.7 \times R_{\mathrm{eff}} \times 10^{22} \mathrm{~m}^{-2} \mathrm{~s}^{-1}$,

where $R_{\text {eff }}$ is the effective radius of the boulder, $\rho$ is the boulder density, $C_{D}$ is the drag coefficient $(=0.47)$, and $c_{g}$ is the vapor sound speed $\left(\approx 350 \mathrm{~m} \mathrm{~s}^{-1}\right)$. Therefore, the formula represents the minimum molecular gas production rate that some local activity, outburst, or event need to at least detach the spherical object from the ground. By way of comparison, El-Maarry et al. (2017) reported for $8.5 \times 10^{23} \mathrm{~m}^{-2} \mathrm{~s}^{-1}$ for jumping boulder 1 , while a theoretical range of $5 \times 10^{21} \mathrm{~m}^{-2} \mathrm{~s}^{-1}$ (Knollenberg et al. 2016) to $1.1 \times 10^{24} \mathrm{~m}^{-2} \mathrm{~s}^{-1}$ (Agarwal et al. 2017) has been reported for some outbursts. During the perihelion passage of mid-to-end 2015 , the peaking $\mathrm{H}_{2} \mathrm{O}$ production was $5.88 \times 10^{20} \mathrm{~m}^{-2} \mathrm{~s}^{-1}$ (Lai et al. 2016). At the peak, the average gas production rate was able to lift centimeter-sized grains, while outbursts could reach the necessary production rate to accelerate meter-sized boulders off the ground.

\footnotetext{
https://docs.scipy.org/doc/scipy/reference/odr.html
}

\section{Local morphological changes}

\subsection{Status before perihelion}

Before perihelion, we have only the color sequence of January 16, 2015, UTC 01:27 showing the deposit at small spatial resolution $\left(\sim 50 \mathrm{~cm} \mathrm{px}^{-1}\right.$, Table 1$)$. The whole sequence displays in long-standing shadow the downhill rough depression bound by the Apis plateau and Anubis in the equatorial reach of the Khonsu region. Nonetheless, in the western corner, shown in Figs. 3b and c (left), we glimpse the High Khonsu bank and part of the Low Khonsu bank, both partially shaded. This particular color sequence has not compressed artifacts in the shaded pixels, that is, pixels with a low signal-to-noise ratio $(\mathrm{S} / \mathrm{N})$, which are generally present when images are data compressed to a certain level ${ }^{8}$. This allows us to perceive indirectly retroilluminated morphological units.

The measured dimensions alongside the tags used in this section and throughout this work are listed in Tables 3 and 2. Both tables contain their effective length, height, volume, and corresponding masses. Because the sharp boundaries of the landmarks are only partially or not fully described by the SPG SHAP7 (Preusker et al. 2017), we equated them to idealized shapes to estimate their volumes, such as cylinders $(\mathrm{C} 1, \mathrm{C} 2, \mathrm{C} 3$, and $\mathrm{C} 4)$, parallelepipeds (C5), trapezoids (F1, C6), ellipsoids (F2, F3), spherical caps (F4), and prisms (F5). Masses were estimated based on the global density of the nucleus $\rho=533 \pm 6 \mathrm{~kg} \mathrm{~m}^{-3}$ (Paetzold et al. 2016; Jorda et al. 2016).

Low bank. In Fig. 3b (left), the partial view of the Low bank shows a terrain that is apparently drier and populated by large blocks. Partially shaded, Patch 1 is a 3-m-deep cavity (C4) situated under a former mass waste that has partially exposed an ice-enriched subsurface. Patch 1, observed to be constantly bluer during $1.5 \mathrm{yr}$, can be considered a perennial feature and will probably remain ice-enriched until the next perihelion passage of 67P (similar long-standing bright features are studied in Oklay et al. 2017 and Deshapriya et al. 2018).

Not far from Patch $1\left(-23.5^{\circ}, 198^{\circ}\right)$, we observe in high resolution the bright spot that survived from March to May 2015. The spot has sustained a flat spectral slope in the visible range (Deshapriya et al. 2016). These features are the only two phenomena of ice-enrichment before perihelion in the Low bank. They are surrounded by a thick dust layer of $100 \mathrm{~m}$ that is $4-5 \mathrm{~m}$ thick, near a shallow cavity (C5).

In the southeastern area of the Low Khonsu bank $\left(-28^{\circ}\right.$ to $-20^{\circ}, 197^{\circ}$ to $-210^{\circ}$ ), we lack a high-resolution image counterpart to detect small morphological changes. The best spatial resolution sequence for this area was only later obtained on May 2, 2015, UTC 15:09, shown in Fig. 3 (left) at $230 \mathrm{~cm} \mathrm{px}^{-1}$. Similarly, the bank is apparently covered by a smooth and dust-like terrain. There, we also observe Patch 1 under better illumination conditions, inside cavity $\mathrm{C} 4$, near the thick dust layer, as has been noted before. From January 16, 2015, on, both features have survived for at least four months. We also highlight the pancake structure $\left(-20^{\circ},-210^{\circ}\right.$; El-Maarry et al. 2016; Deshapriya et al. 2016), a remarkable landmark of three superimposed slabs in Khonsu.

High bank. In Fig. 3c (left), we note the High bank, which contains an 8-m-high mound (F4) and an extended blue patch

8 https://pds.jpl.nasa.gov/ds-view/pds/viewProfile. j sp?dsid=R0-C-OSIWAC-3-ESC1-67PCHURYUMOV-M12-V1 . 0 
(a)
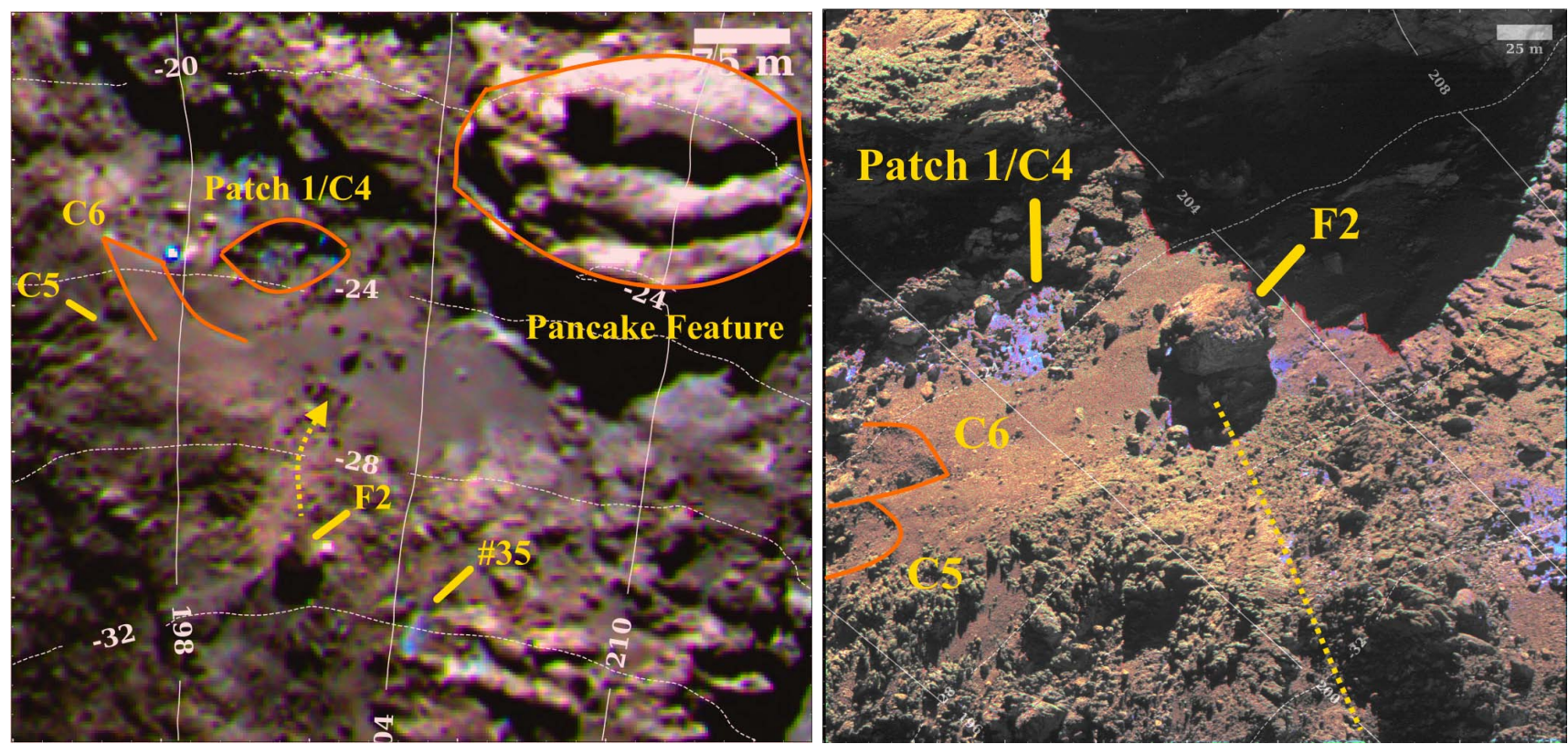

(b)
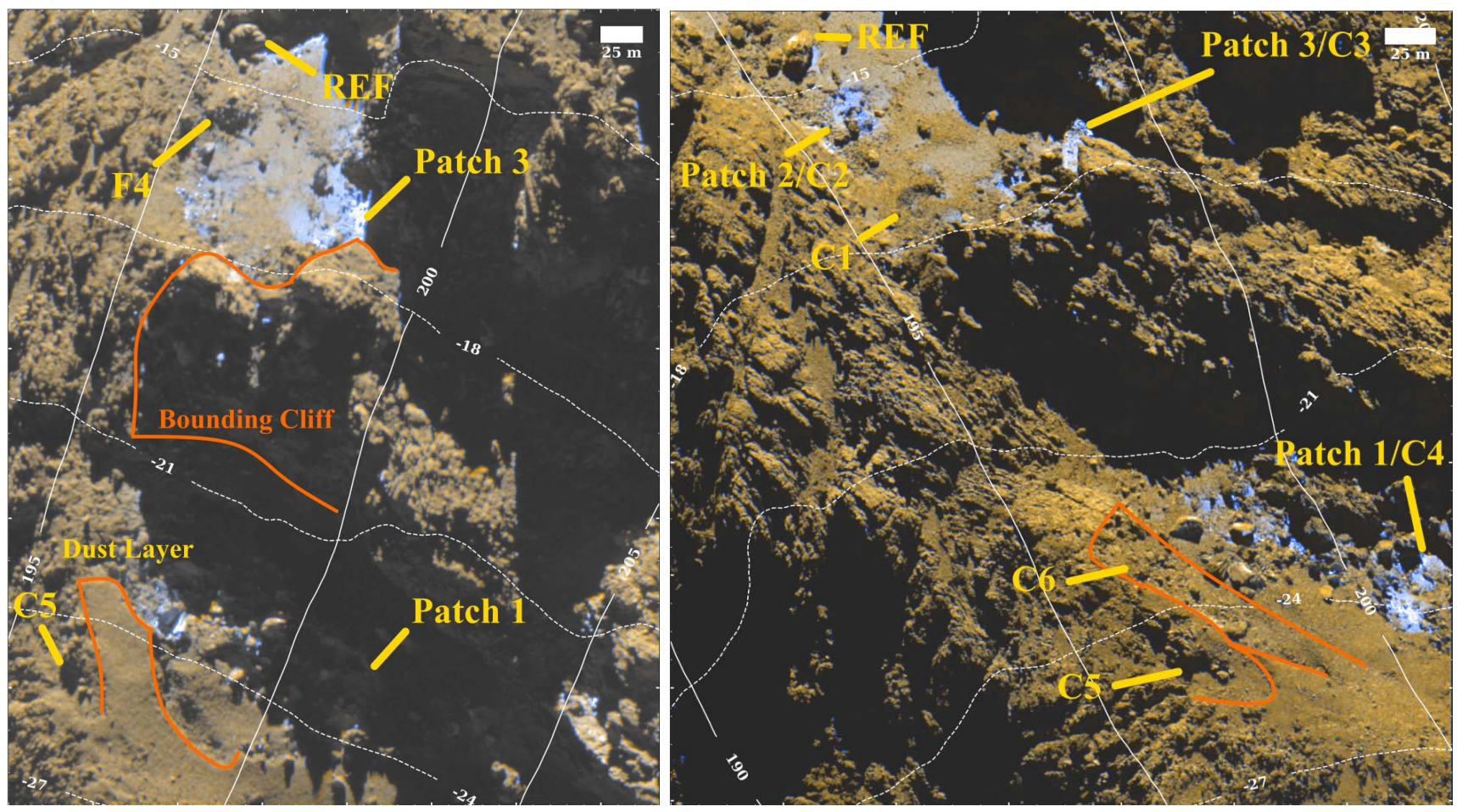

Fig. 3. Morphological changes after perihelion passage. OSIRIS RGB composites obtained in the final Rosetta multi-instrumental campaign of 2016 next to other OSIRIS RGB composites acquired before perihelion passage of July-October 2015. Labels used here are the same as labels in Tables 3 and 2 and throughout the text. Qualitatively, RGB bluer terrains correspond to a different gradient of the flat VIS-to-NIR spectral slope. In Sect. 5 we present the quantitative estimation for the ice-enriched units. (a) May 2, 2015, UTC 15:09 (F41-F23-F24, left) and July 16, 2016, UTC 19:22 (F41-F23-F24, right). Low Khonsu bank. The symbol " $\leftarrow--$ " represents the displacement of jumping boulder 1. "35" indicates the source of event 35 described in Sect. 6. (b) January 16, 2015, UTC 01:27 (F22-F22-F24, left) and June 25, 2016, UTC 18:02 (F22-F22-F24, right). Partial view of the High and Low Khonsu bank.

(Patch 3) over a terrain of smooth texture. In the same figure and slightly north of it, beginning at the 10-m-wide REF boul$\operatorname{der}\left(-14.5^{\circ}, 195.5^{\circ}\right)$ and stretching for $\sim 200 \mathrm{~m}$ up to the base of the equatorial cliff at $-10^{\circ}$ latitude, a high-mass wall (F1) of $10 \mathrm{~m}$ height is visible. $\mathrm{F} 1$ is also visible in Fig. 5a at more favorable illumination, showing a 30 -m-wide retreat at its west side $\left(-12.5^{\circ}, 194^{\circ}\right)$ that exposed another enriched terrain underneath. At its east side $\left(-12^{\circ}, 198^{\circ}\right)$, two 20 -m-sized boulders are found clustered at the base of an ancient cliff collapse, on the top of the wall.

\subsection{Status after perihelion}

Sequences acquired during the final multi-instrumental observations of June-July 2016 allow us to investigate the Khonsu bank at a decimeter scale $\left(0.15-0.3 \mathrm{mpx}^{-1}\right)$ after perihelion. In this 
(c)

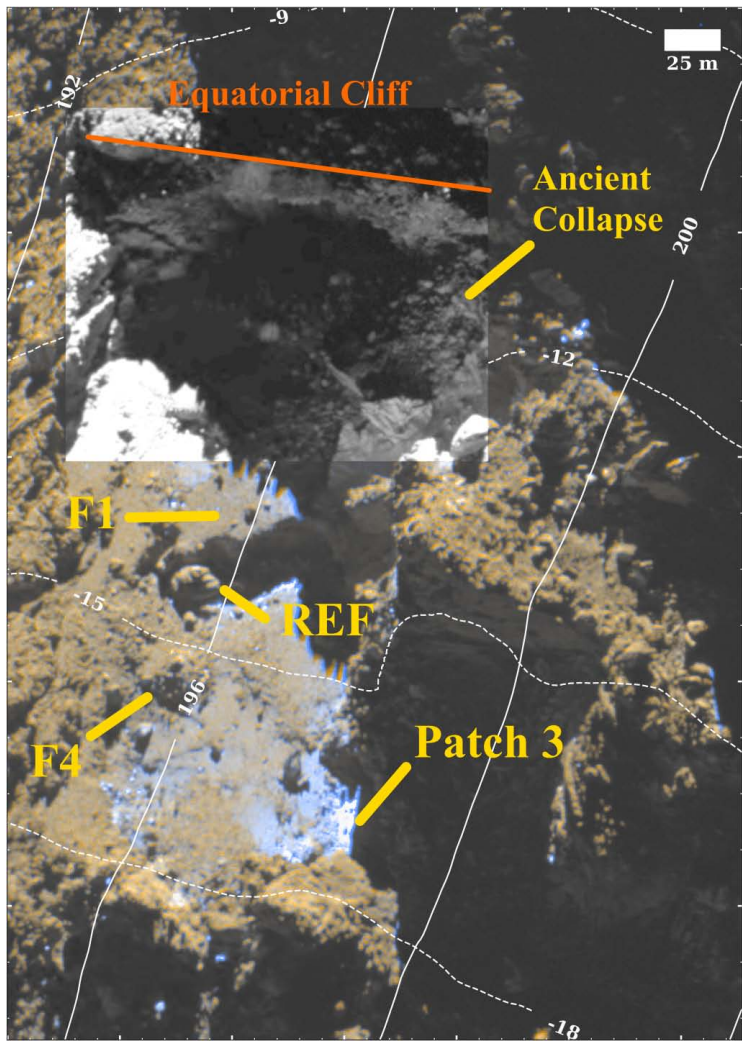

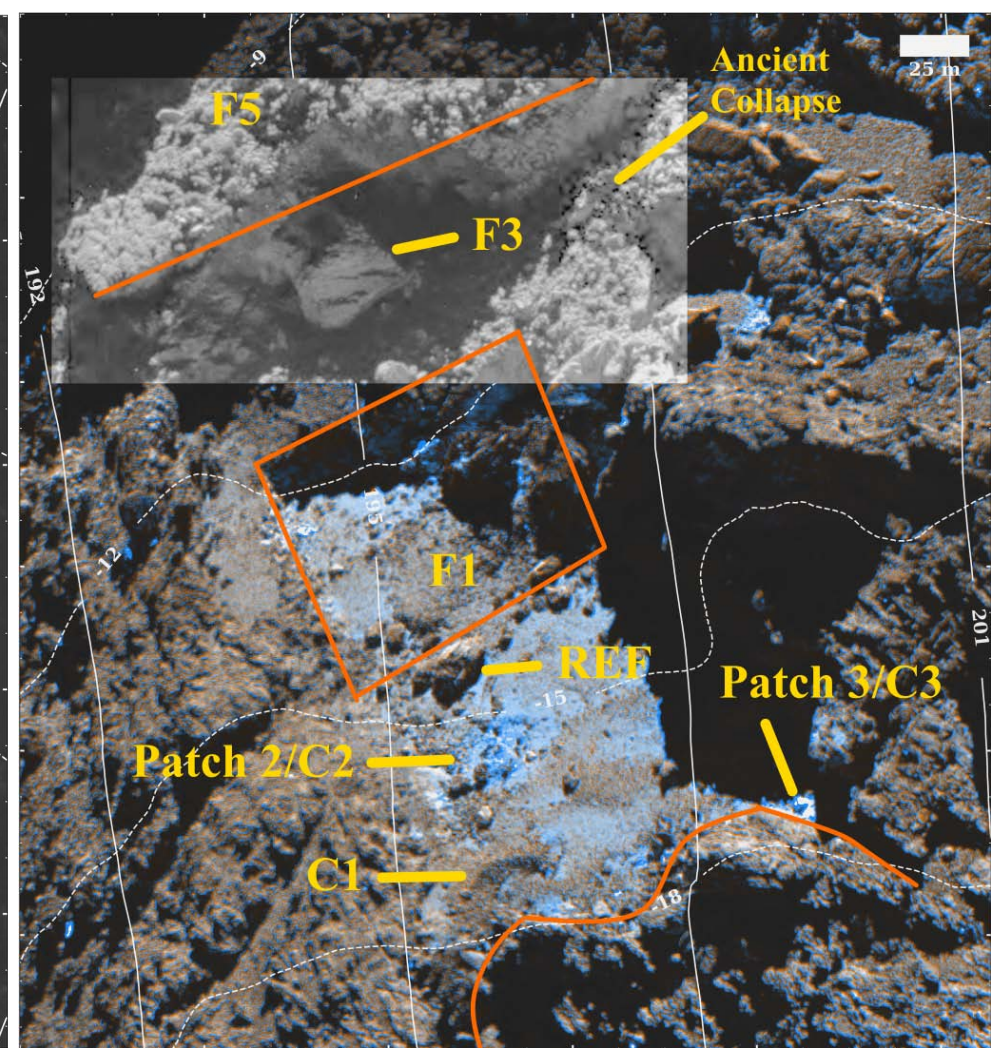

Fig. 3. continued. (c) January 16, 2015, UTC 01:27 (F22-F22-F24, left) and June 25, 2016, UTC 06:09 (F22-F22-F24, right). The High Khonsu bank. In the upper panels we substitute the shaded area where jumping boulder 2 was located for the contrast-stretched counterparts. The orange perimeter roughly marks the locus of the retreating wall (F1).

context, the dimensions of landmarks at a scale of a few meters can be estimated to an uncertainty better than $20 \%$. In particular, the sequences on June 25, 06:09, June 25, 18:02, July 2, 07:57, July 9, 08:28, and July 16, 19:22, have the highest spatial resolution obtained for the Khonsu region $\left(\sim 16-35 \mathrm{~cm} \mathrm{px}^{-1}\right)$.

Low bank. Ten months after perihelion, the OSIRIS color sequences revealed several surface changes that have taken place between mid-to-end 2015. Along with the RGB from May 2, 2015, 15:09, the color sequence of July 16, 2016, 19:22 (Fig. 3a, right) provides the highest resolution image achieved for Khonsu, displaying the precise frame of Patch 1 and jumping boulder $1(\mathrm{~F} 2)$. F2 is a 50-m-sized boulder that rolled downhill from $\left(-30^{\circ},-201^{\circ}\right)$ at a distance of $\sim 140 \mathrm{~m}$ and left no subsurface ice-enriched trail (El-Maarry et al. 2017). Patch 1 shows a remarkable smooth blue terrain inside a cavity $(\mathrm{C} 4)$, surrounded by a mass waste of meter-sized boulders. We have counted a total of 825 boulders and fit the boulder-size cumulative distribution (Fig. 4). The wasting of Patch 1 shows a power-law index of $-1.6 \pm 0.01$ for the $1.2-10 \mathrm{~m}$ size range, a flat distribution when compared to -3.6 for the entire nucleus (Pajola et al. 2015) and to -3.1 for the entire Khonsu area (Deshapriya et al. 2016). This implies that the mass waste is dominated by large blocks of comparable size to the depth of $\mathrm{C} 4$. Flat distributions also indicate non-negligible tensile strength in the top $3 \mathrm{~m}$ of depth. The lack of large debris at the base of C4/Patch 1 indicates that the area experienced a gas production that was energetic enough to scatter the large blocks to the surroundings. The largest members of $\sim 6 \mathrm{~m}$ radii in the boulder-size distribution of Fig. 4 may indicate that $\mathrm{C} 4$ was excavated in the past at a local top gas production rate of $3.4 \times 10^{23} \mathrm{~m}^{-2} \mathrm{~s}^{-1}$.



Fig. 4. Boulder-size cumulative distribution associated with the wasting at Patch 1 from images of June 25, 2016, UTC 18:02 and July 7, 2016, UTC 19:22. Logarithmic cumulative number normalized by total boulder surface, in $\mathrm{km}^{2}$, as logarithmic function of boulder size, in meters. Completeness for $>1.2 \mathrm{~m}$ in size. Two power-law indices are calculated: $-1.6 \pm 0.01$ for $1.2-10 \mathrm{~m}$; and $-1.8 \pm 0.14$ for $10-15 \mathrm{~m}$. The average error in size is estimated at $0.37 \mathrm{~m}$.

It roughly falls within the range of gas production for $67 \mathrm{P}$ outbursts. 
Table 2. Property of large displaced or missing landmarks identified after perihelion.

\begin{tabular}{|c|c|c|c|c|c|c|c|}
\hline Feature & $\varphi\left({ }^{\circ}\right)$ & $\lambda\left(\left(^{\circ}\right)\right.$ & $\begin{array}{l}\text { Eff. lengths } \\
(\mathrm{m})\end{array}$ & Eff. height (m) & Eff. volume $\left(\mathrm{m}^{3}\right)$ & Mass (kg) & Context \\
\hline $\mathrm{F} 1$ & -12.3 & 194.8 & $\begin{array}{r}102.8 \pm 2.6 \\
\times 64.6 \pm 1.1 \\
\times 100.4 \pm 1.1\end{array}$ & $9.3 \pm 0.1-14.3 \pm 1$ & $(1.0 \pm 0.2) \times 10^{5}$ & $(5.3 \pm 1) \times 10^{7}$ & Retreating wall \\
\hline $\mathrm{F} 2$ & -26.3 & 202.8 & $56.4 \pm 0.8$ & $48.5 \pm 0.9$ & $(8.1 \pm 0.2) \times 10^{4}$ & $(4.3 \pm 0.1) \times 10^{7}$ & Jumping boulder 1 \\
\hline $\mathrm{F} 3$ & -10.6 & 195.0 & $50.6 \pm 0.8$ & $30.4 \pm 4$ & $(4.1 \pm 0.5) \times 10^{4}$ & $(2.2 \pm 0.3) \times 10^{7}$ & Jumping boulder 2 \\
\hline $\mathrm{F} 4$ & -15.3 & 195.7 & $26 \pm 7$ & $7.9 \pm 0.3$ & $(2.6 \pm 0.2) \times 10^{3}$ & $(1.4 \pm 0.1) \times 10^{6}$ & Mound/Patch 2 \\
\hline F5 & -10.6 & 195.0 & $\begin{array}{r}190.4 \pm 7 \\
\times 31.6 \pm 7\end{array}$ & $53.7 \pm 2$ & $(1.6 \pm 0.4) \times 10^{5}$ & $(8.6 \pm 0.2) \times 10^{7}$ & $\begin{array}{l}\text { Deformation/excavation } \\
\text { of equatorial cliff }\end{array}$ \\
\hline
\end{tabular}

Notes. Features and their equivalent geometrical bodies for estimating their volumes: F1 to trapezoid; F2, F3 to ellipsoid; F4 to spherical cap; F5 to prism.

Table 3. Property of cavities measured from ToO image sequences.

\begin{tabular}{cccccccc}
\hline \hline Cavities & $\varphi\left(^{\circ}\right)$ & $\lambda\left(^{\circ}\right)$ & Eff. lengths $(\mathrm{m})$ & Eff. height $(\mathrm{m})$ & Eff. volume $\left(\mathrm{m}^{3}\right)$ & Mass $(\mathrm{kg})$ & Context \\
\hline C1 & -17.2 & 196.1 & $25.8 \pm 1.5$ & $4.8 \pm 1$ & $(2.5 \pm 0.6) \times 10^{3}$ & $(1.3 \pm 0.3) \times 10^{6}$ & Dry \\
C2 & -15.6 & 196.0 & $18.8 \pm 4.7$ & $2.8 \pm 0.4$ & $(7.7 \pm 4) \times 10^{2}$ & $(4.1 \pm 2) \times 10^{5}$ & Patch 2 \\
C3 & -16.8 & 199.4 & $16.3 \pm 0.8$ & $1.4 \pm 0.2$ & $(2.8 \pm 1) \times 10^{2}$ & $(1.5 \pm 0.5) \times 10^{5}$ & Patch 3 \\
C4 & -23.1 & 201.8 & $62.8 \pm 1.95$ & $3.2 \pm 0.4$ & $(9.4 \pm 0.8) \times 10^{3}$ & $(5.2 \pm 0.4) \times 10^{6}$ & Patch 1 \\
C5 & -25.2 & 197.0 & $87.9 \pm 0.8 \times 27.6 \pm 0.9$ & $5.9 \pm 0.7$ & $(1.4 \pm 0.2) \times 10^{4}$ & $(7.6 \pm 0.9) \times 10^{6}$ & Dry \\
C6 & -24.1 & 198.0 & $105.8 \pm 6.0 \times 41.8 \pm 5.7$ & $10 \pm 0.3 / 14 \pm 0.8$ & $(5.3 \pm 0.8) \times 10^{4}$ & $(2.8 \pm 0.4) \times 10^{7}$ & $\begin{array}{c}\text { Dry (thick } \\
\text { dust layer) }\end{array}$ \\
\hline
\end{tabular}

Notes. C1, C2, C3, and C4 exhibit a roughly cylindrical shape, C5 is equated to a parallelepiped, and C6 to a trapezoid due to mismatches in height from one extremity to another.

Moving closer to the equator, we compared the sequences acquired on June 25, 2016, UTC 18:02 to January 16, 2015, UTC 01:27 (Fig. 3b) to continue tracking morphological changes in the boundary between the High and Low banks. The first remarkable change is the formation of cavity $\mathrm{C} 6$, where the thick dust layer was previously located. C6 corresponds to a vanishing mass of $(2.8 \pm 0.4) \times 10^{7} \mathrm{~kg}$ that left no sign of ice enrichment at its bottom. Near this feature $\left(-20^{\circ}, 196^{\circ}\right)$, some dust cover has also been removed, exposing a cracked consolidated material underneath.

High bank. Crossing the bounding cliff at $-20^{\circ}$ latitude, we find the southern part of the high bank the location of most of the activity detected by Vincent et al. (2016b) and Deshapriya et al. (2018) and also gas emissions (Laeuter et al. 2019). The dust cover of this area was partially removed, as we observe a clear change in roughness. The removal was gentle, the scattered boulders were not displaced from their original position. Similarly to Patch 1, we still detected ice-enrichment in C3/Patch 3, suggesting a year-long survival.

Near the $\sim 10$-m-high western boundary (rough scarp), we note the formation of two more cavities ( $\mathrm{C} 1$ and $\mathrm{C} 2$ ), both $\sim 20 \mathrm{~m}$ wide and 3-5 m deep. While $\mathrm{C} 1$ was apparently dry, $\mathrm{C} 2$ exhibited signs of ice-enrichment in a smooth terrain (Patch 2). $\mathrm{C} 2$ formed where the 8-m-high mound (F4) was once located. Interestingly, both features were $30 \mathrm{~m}$ apart and experienced similar seasonal shading from the Apis plateau and the rough scarp, but they still show different colors. The masses of the two cavities are on the order of $10^{5}-10^{6} \mathrm{~kg}$. The power-law index of the boulder-size cumulative distribution for the environment of Patch 2 (244 counts, complete between 1.2 to $3 \mathrm{~m}$ ) is
$-2.73 \pm 0.01$, much steeper than for Patch 1 . This likely indicates that the top layers are far more brittle than layers near cliffs.

Retreating wall in the High bank. Finally, in the equatorial reach of the High bank, as seen in Fig. 3c, we observe the most dramatic changes. The most striking change is the receding of the high-mass wall (F1), a landmark that has been noticed before by Deshapriya et al. (2016). We note that area similar to a trapezoid of 64.6-100.4 m wide, $102.8 \mathrm{~m}$ length, and 9.314.3 high has vanished (Table 2). As these 53000 tons of mass sublimated, two 20-m-sized boulders fell onto the bottom of the deposit (Fig. 5c). On one of the two boulders, part of its surface scratched and an ice-enriched spot was revealed that survived for more than six months (Deshapriya et al. 2016). Through a search of the OSIRIS image database, we were able to narrow down the removal of feature F1 to have occurred between August 1, 2015, UTC 08:00 (Fig. 5b) and October 31, 2015, UTC 22:49. As of this date, the wall had completely retreated to the same length as observed in Fig. 3c. A boulder-size counting on June 25, 2016, 06:09 image at the regime of 1-15 $\mathrm{m}$ for 404 boulders was fit by a power-law distribution of $-2.09 \pm 0.01$, similar to the Neck region (-2.2, Pajola et al. 2015). This might be an indication of strong consolidation of the boulders that rolled after F1 vanished and/or a certain lack of blocks smaller than $10 \mathrm{~m}$ as components of F1's interior.

Jumping boulder 2 in the High bank. In contact with the equatorial cliff and lying over the part of scarp F1 that has not receded, we note the appearance of another 50-m-wide boulder (F3) that was not detected from January 16, 2015, until August 1, 2015, UTC 08:00 (Fig. 5b). In Fig. 3c we have stretched the F22 
(a)



(b)

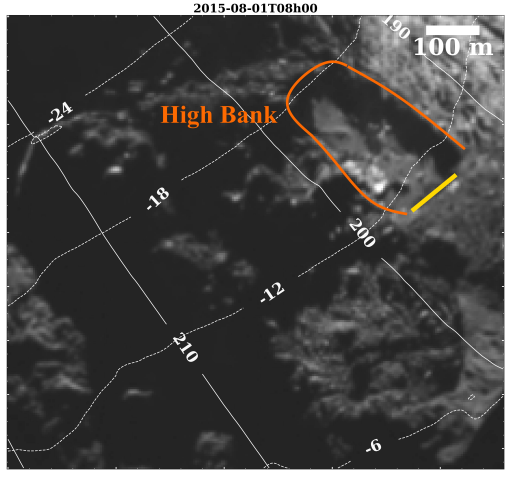

(c)

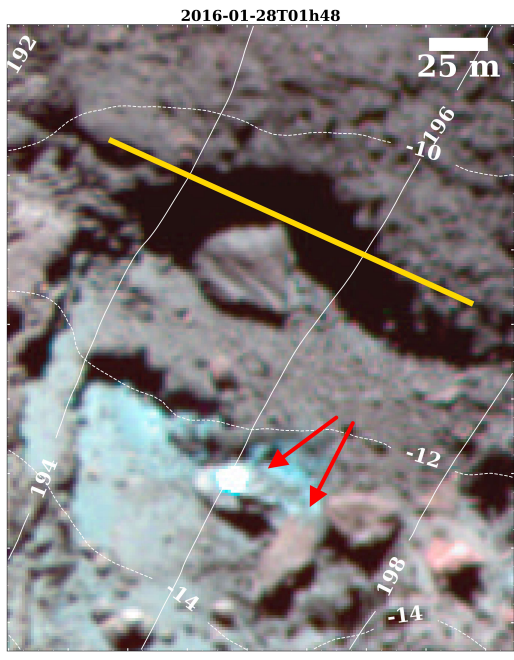

Fig. 5. Consequence of the morphological changes that took place in the High Khonsu bank during the perihelion passage. (a) May 2, 2015, UTC 15:09 (F41-F23-F24); (b) August 1, 2015, UTC 08:00 (F22); (c) and January 1, 2016, UTC 01:48 (F41-F23-F24). Description: (a) Landmark F1 (retreating wall) and the two 20-m-sized boulders near an ancient cliff collapse observed at better illumination conditions. (b) The High bank observed from an S/C distance of $215 \mathrm{~km}$. Landmark F1 is still observable, and no deformation (yellow line) of the equatorial cliff is detected. (c) Jumping boulder 2 under better illumination conditions. F1 has vanished, and two 20-m-sized boulders are now found to have fallen onto the deposit. Deformation caused by jumping boulder 2 is marked by a yellow line.

image contrast of the F3 area for better visualization. In Fig. 5c we can observe this new jumping boulder in a better framing. Visual inspection of the images of Khonsu before perihelion does not show any object of similar size over the neighboring cliffs, over the Apis plateau, or on top of the ancient cliff collapse nearby. Therefore, we can at least rule out that F3 rolled down from any obvious or nearby surroundings. Additionally, we note that the appearance of F3 is connected to a deformation and/or mass loss into the nearby cliff when we compare Figs. 5b and c. This deformation or excavation (F5) corresponds to a mass of $8.6 \times 10^{7} \mathrm{~kg}$ a ratio of 3.9 to the mass of F3. In Sect. 7.1.5 we investigate the possible scenarios for the origin of jumping boulder 2 .

\section{Spectral slope in the visible range}

Spectral slopes in the visible range (VIS) were calculated by linear regression of each pixel for all filters in the sequence, which was normalized to NAC F22 filter $(649 \mathrm{~nm})$ instead of the usual NAC F23 (535 nm) adopted in the literature. This was done because in the color sequence of January 16, 2015, UTC 01:27, the NAC F23 band is lacking.

To access the degree of ice enrichment in the Khonsu bank, we selected all pixels with albedos $5 \%$ higher and spectral slopes $5 \%$ bluer than the surface average for same given phase angle in the observations of January 16, 2015, and June 25/July 2, 2016 (see Table 1). The presence of ice grains causes the VIS spectral slope to become flat and the albedo to rise nonlinearly with respect to the ice abundance (Pommerol et al. 2015; Fornasier et al. 2016; Barucci et al. 2016; Oklay et al. 2016a; Raponi et al. 2016). Figure 6 shows the color-coded normal albedo $A_{0, \lambda=649}^{\prime}$ and VIS spectral slope for January 16, 2015, $\left(r=2.5\right.$ AU, pre-perihelion, $\left.\alpha=97^{\circ}\right)$ and June 25/July 2, 2016, $\left(r=3.2\right.$ AU, post-perihelion, $\left.\alpha=72^{\circ}-93^{\circ}\right)$ overplotted on their respective RGB composites.

The color sequence of January 16, 2015, seven months before the perihelion passage, shows a High Khonsu bank that is partially shaded and populated with areas that are highly enriched in ices (Fig. 6). The regional $A_{0, \lambda=649}^{\prime}$ is as high as $7.9 \%$ even disregarding the opposition effect, whereas $67 \mathrm{P}$ has a global albedo of $6.8 \%$ (Fornasier et al. 2015; Hasselmann et al. 2017) when all photometric effects are otherwise considered.

The entire westernmost area, where 16 months later we observe the appearance of $\mathrm{C} 1$ and $\mathrm{C} 2 / \mathrm{Patch} 2$, is mildly bluer $(\sim 9-12 \% / 100 \mathrm{~nm})$ and brighter $(>16 \%)$. The spectral slope gradient in the smooth terrain becomes bluer near shadow lines. The brightest and bluest zones are very near to the scarps, where they are subjected to shading for a long period of time. We locate three of such zones: two situated in the surroundings of the retreating wall $\left(\mathrm{F} 1 ;-12.2^{\circ}, 195^{\circ}\right.$ and $\left.-14.5^{\circ}, 197^{\circ}\right)$, and one that we named Patch $3\left(-16.8^{\circ}, 198^{\circ}\right)$, a particularly blue area concealed by cliffs that survived until July 2016 (Fig. 6b). Patch 3 extends for about $50 \times 30 \mathrm{~m}^{2}$, but its bluest corner is constrained at $1.7 \mathrm{~m}^{2}$. This bluest corner clings to the shadow line and shows an VIS slope of $-1 \pm 3 \% / 100 \mathrm{~nm}$ and $A_{0}^{\prime}>56 \pm 3 \%$. When we consider that coarse water ice has $A_{0}=99 \%$ and the neutral VIS spectrum, this corner must bear a component of dark comet-average material of no higher than $0.1 \%$ to preserve its flat spectral slope according to ice+carbon+tholin laboratory experiments (Jost et al. 2017) and bright spot studies with VIRTIS-M (Raponi et al. 2016).

To obtain an insight into the conditions that the High bank was exposed to during this epoch, we simulated the irradiance and shadow casting using NAIF SPICE and OASIS for one cometary rotation during January 16, 2015. In Fig. 7 we show simulated irradiance frames for three given UTC hours around mid-day, close to the observation time. Until mid-day (Fig. 7a), the region was concealed by shadow casting from the Apis plateau above. The shadow line moved progressively until 1 UTC hour later, when the color sequence of January 16, 2015, UTC 01:27 was obtained and the bluer VIS spectral slope 


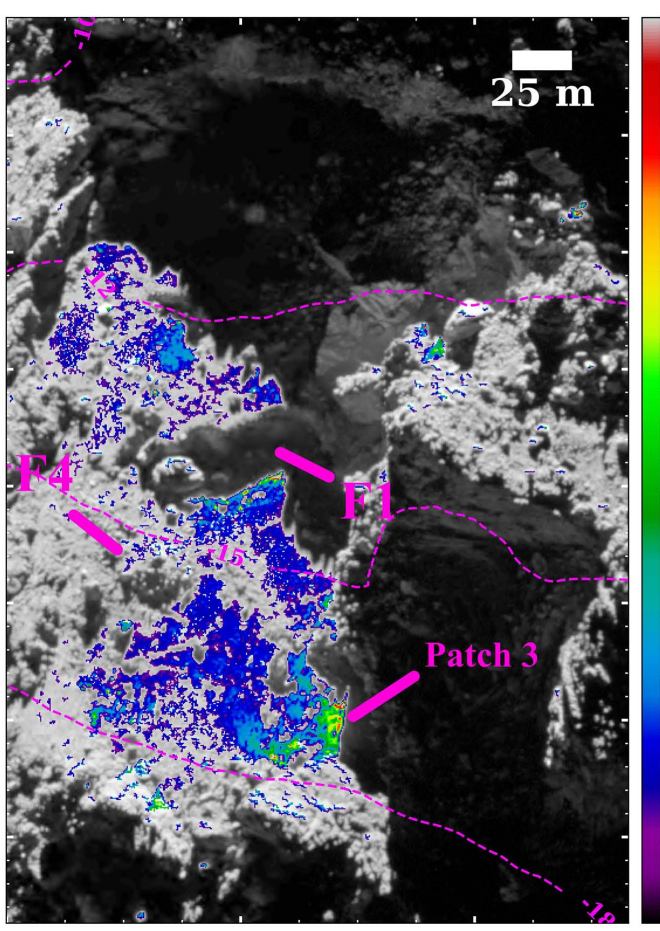



$-24.0$

$\mathrm{i}=80.5 \mathrm{e}=30.8 \mathrm{ph}=97.3 \mathrm{A0}=7.9$ theta $=45.0$ (No SHOE)

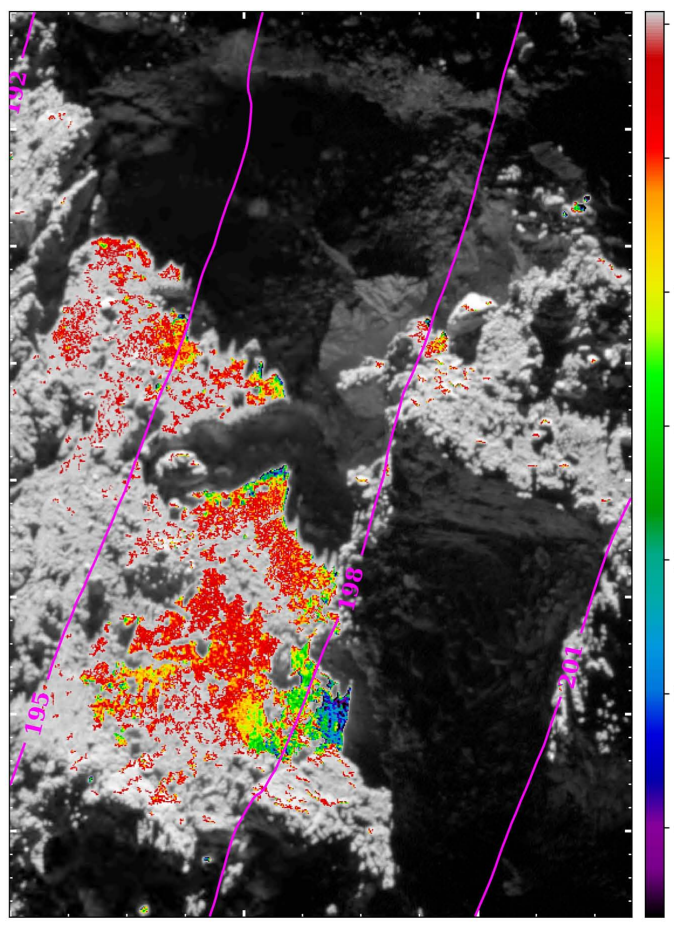

15.0

12.0

$9.0 \underset{c}{\infty}$

6.0 $\frac{\pi}{8}$

$3.0 \stackrel{2}{2}$

0.0

12.0

$-3.0$

$$
\begin{gathered}
\mathrm{i}=80.1 \mathrm{e}=24.8 \mathrm{ph}=96.6 \mathrm{A0}=6.4 \\
\text { theta }=45.0(\text { No SHOE })
\end{gathered}
$$
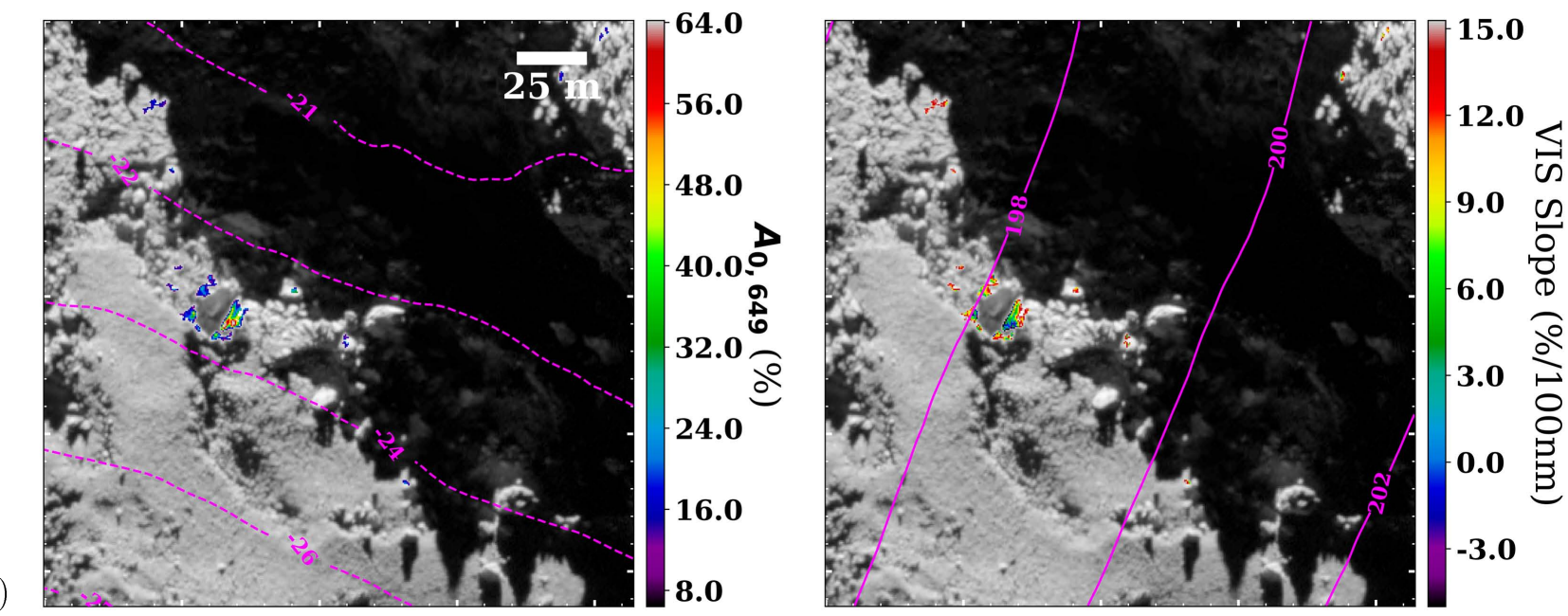

Fig. 6. NAC F22 normal albedo and VIS spectral slope of Khonsu bank before and after perihelion passage. Normal albedo and VIS slope are overplotted on their RGB composites. (a) January 16, 2015, UTC 01:27 divided into two panels that show the High and Low banks. The median VIS spectral slope is $15.95 \% / 100 \mathrm{~nm}$.

gradient was detected. The High bank continued to be illuminated for 1:30 UTC hours until it was covered in shadows for the next $\sim 9$ UTC hours. The insolated surface temperature is approximately $230 \mathrm{~K}$ at $2.53 \mathrm{AU}$ (Fig. 2), which is high enough to sublimate water ice and super-volatiles. Based on this short cycle of insolation and the surface temperature, we consider that frost, surface ices that recondens from immediately below, is the mechanism that is most compatible with the observed blue color gradient that follows the same direction as the shadow lines. Frost has been proposed by Feaga et al. (2007) and Prialnik et al. (2008) and has recently been observed by De Sanctis et al. (2015) in the Hapi region and also by
Fornasier et al. (2016) in the Imhotep region. There, blue features disappeared according to insolation and topography, and apparently moved following the shadow casting speed. This phenomenon is highly ephemeral and superficial. Fornasier et al. (2016) estimated a layer of only tens of micrometers and tens of grams per squared meter. This indicates that the High bank suffered a recurring cycle of ice redeposition and sublimation in the surface that petered out as the local sub-solar latitude decreased toward the southern regions. This specific condition might be related to the activity mechanism that led to the appearance of cavities in the High bank. Activity mechanisms are discussed in Sect. 7.3. 

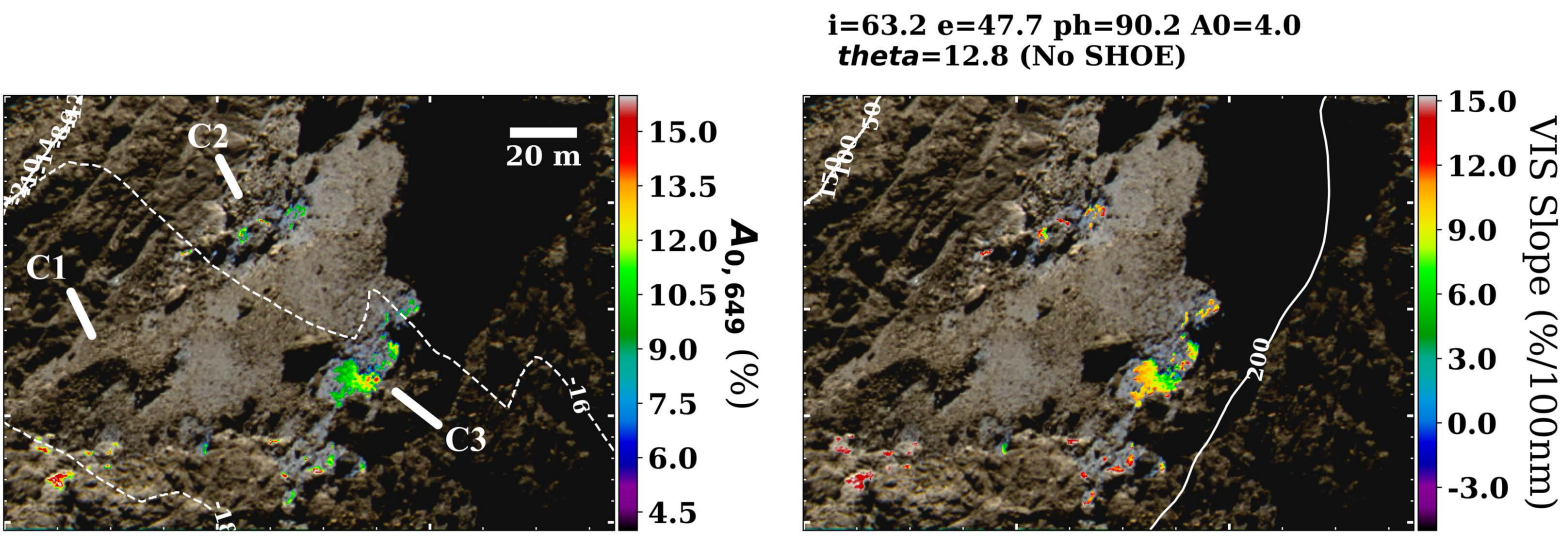

$\mathrm{i}=66.5 \mathrm{e}=35.5 \mathrm{ph}=92.9 \mathrm{A0}=3.6$ theta $=1.0$ (No SHOE)
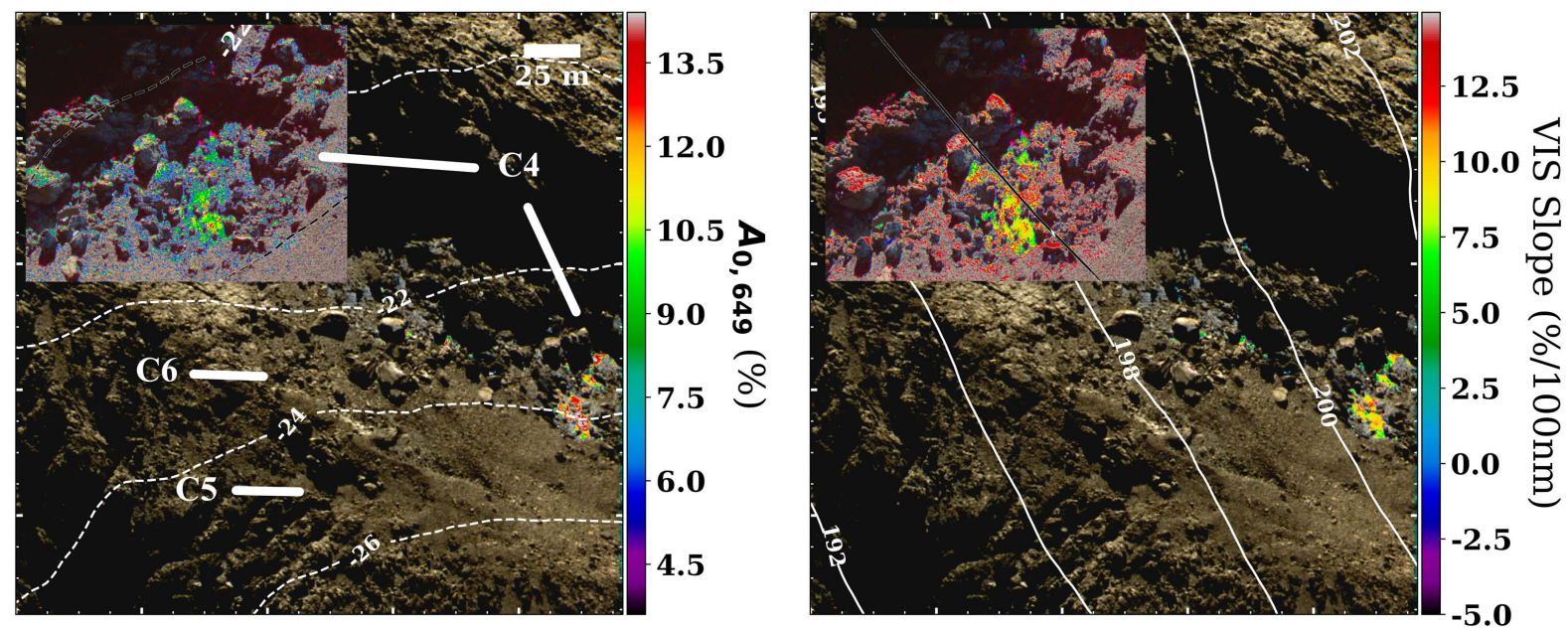

Fig. 6. continued. Panel b: July 2, 2016, UTC 07:57 (top panels, High bank) and June 25, 2016, UTC 18:02 (bottom panels, High and Low banks). The median VIS spectral slopeis $15.90 \% / 100 \mathrm{~nm}$.

The visible part of the Low bank (Fig. 6a, bottom) is notably drier than the High bank. Only one very bright blue spot $\left(A_{0}^{\prime}>65 \%\right)$ is detected. This blue feature lies below a $5-\mathrm{m}$ boulder $\left(-23^{\circ}, 198.2^{\circ}\right)$, and the spectrotemporal evolution was reported by Deshapriya et al. (2016).

During the arget of opportunity color sequences in 2016 (Fig. 6b), we observed that the extent of ice-enriched zones dramatically decreased. These zones were found to be nested in three blue patches. Patch 3 is not larger than $20 \mathrm{~m}$, while Patch 2 exhibits only a few spectrally bluer pockets. All patches are situated inside depressions and exhibit a smooth texture at a decimeter scale. Patch 3, in particular, has undergone 1-2 m (C3) of dust layer removal with a similar aspect to the process reported by Groussin et al. (2015) in the large smooth terrain of the Imhotep region and has become spectrally redder through this alteration $(\sim 3-6 \% / 100 \mathrm{~nm}$ for the bluest core). The regional normal albedo has also decreased by $50 \%$ for both the High and Low banks.

\section{Local activity and dust mass}

We visually inspected all color sequences listed in Table 1 to measure every event originating from the Khonsu bank, and we manually selected the spot of maximum RADF (all pixels below $P(R A D F)>95 \%$ with respect to the maximum intensity of the dust plume) as their most probable origin. We note that all sequences of August 1, 2015, were obtained when the base of the bank was still shaded. This is the same condition for the first color sequence of December 13, 2015 (01:13), while the outburst source is partially visible on the second sequence (02:13). The source location of the outburst on September 14, 2015, was constrained by Vincent et al. (2016b) through triangulation of the two outburst components. In total, we detected a total of 36 events ranging from three massive outbursts to several mild plumes. The properties of the plumes are summarized in Table 4. We refer to this table for the numbering of events reported in this section and throughout this paper.

As the plumes were steady and stationary through all NAC filters, we were also able to measure their VIS spectral slope (here normalized to the NAC F23 filter, $535 \mathrm{~nm}$, see Sect. 5). However, to avoid major spectral contamination of the inner coma in between the plume and the detector, or from the backlit surface of the nucleus, we report only the integrated color of pixels at the maximum intensity spot. The average VIS spectral slope is $10.36 \pm 3 \% / 100 \mathrm{~nm}$, similar to the quiescent coma measured by VIRTIS-M (9-12\%/100 nm, Rinaldi et al. 2016). The plumes are also very heterogeneous, some (numbers 9, 15, and 23 in Table 4) present much bluer VIS spectra, indicating a significant component of nanometer and/or icy grains. Other events, such as event $8-10$, are relatively redder, showing otherwise a predominance of larger-than-wavelength grains. Interestingly, from the same site as event 10 arises event 5, one hour earlier, which has a color that is $50 \%$ bluer. This indicates that as the Sun illuminates the site $\left(2: 00 \mathrm{~h}_{\text {comet }}\right.$ to $\left.4: 00 \mathrm{~h}_{\text {comet }}\right)$, the production 
Table 4. Table of properties of all activity detected on the Khonsu bank and its proximities.

\begin{tabular}{|c|c|c|c|c|c|c|c|}
\hline ID & Sequence & lat $\left({ }^{\circ}\right)$ & $\operatorname{lon}\left(^{\circ}\right)$ & Local time $\left(\mathrm{h}_{\text {comet }}\right)$ & VIS slope $(\% / 100 \mathrm{~nm})$ & $R A D F_{\max }(\%)$ & Dust mass $(\mathrm{kg})$ \\
\hline 0 & 2015-08-01, 08:43 & -14.1 & 196.1 & $21: 00$ & 7.2 & 0.087 & 500 \\
\hline 1 & 2015-08-01, 10:51 & -11.1 & 196.4 & 01:05 & 9.8 & 0.611 & \\
\hline 2 & " & -10.4 & 194.6 & " & 8.9 & 0.448 & 50820 \\
\hline 3 & " & -11.8 & 195.9 & " & 10.5 & 0.478 & \\
\hline 4 & 2015-08-01, 11:21 & -10.8 & 195.0 & 02:05 & 12.2 & 0.266 & \\
\hline 5 & $\prime \prime$ & -11.0 & 196.1 & $\prime \prime$ & 7.8 & 0.204 & 980 \\
\hline 6 & ' & -12.2 & 196.8 & " & 8.1 & 0.219 & 3015 \\
\hline 7 & 2015-08-01, 11:51 & -12.7 & 196.1 & 03:05 & 8.5 & 0.192 & 195 \\
\hline 8 & " & -11.2 & 196.2 & $\prime \prime$ & 14.9 & 0.140 & 2870 \\
\hline 9 & " & -10.8 & 193.9 & " & 6.8 & 0.183 & 185 \\
\hline 10 & 2015-08-01, 12:21 & -11.0 & 195.8 & 04:00 & 17.3 & 0.168 & \\
\hline 11 & " & -10.8 & 194.1 & " & 15.9 & 0.151 & 1940 \\
\hline 12 & 2015-08-01, 20:55 & -13.9 & 196.3 & $20: 40$ & 9.9 & 0.155 & 620 \\
\hline 13 & " & -12.8 & 195.3 & $\prime \prime$ & 15.1 & 0.057 & \\
\hline 14 & " & -12.0 & 195.2 & $\prime \prime$ & 13.5 & 0.046 & 415 \\
\hline 15 & 2015-08-01, 21:25 & -13.7 & 196.1 & $21: 40$ & 6.9 & 0.058 & 725 \\
\hline 16 & $\prime \prime$ & -12.9 & 195.1 & $\prime \prime$ & 12.7 & 0.034 & 725 \\
\hline 17 & " & -11.1 & 195.0 & " & 14.4 & 0.066 & \\
\hline 18 & " & -10.6 & 193.8 & " & 11.5 & 0.066 & 280 \\
\hline 19 & 2015-08-01, 21:55 & -13.9 & 197.4 & $22: 45$ & 14.8 & 0.065 & 650 \\
\hline 20 & $\prime \prime$ & -11.1 & 195.7 & $22: 30$ & 8.3 & 0.205 & 1615 \\
\hline 21 & $2015-08-01,22: 25$ & -20.0 & 205.5 & $00: 10$ & 7.6 & 0.047 & 595 \\
\hline 22 & " & -11.1 & 196.1 & $23: 35$ & 11.1 & 0.141 & 3640 \\
\hline 23 & " & -6.1 & 189.3 & $23: 05$ & 6.6 & 0.013 & 140 \\
\hline 24 & 2015-08-01 22:55 & -11.1 & 196.4 & 00:30 & 11.1 & 0.077 & \\
\hline 25 & " & -11.9 & 197.1 & " & 8.9 & 0.059 & 810 \\
\hline 26 & " & -22.6 & 201.0 & $00: 55$ & 3.3 & 0.014 & \\
\hline 27 & " & -23.3 & 200.7 & " & 7.8 & 0.013 & 150 \\
\hline 28 & $\prime \prime$ & -22.9 & 203.9 & 01:00 & 9.4 & 0.010 & \\
\hline 29 & $2015-08-01,23: 25$ & -11.5 & 196.9 & $01: 30$ & 8.7 & 0.038 & \\
\hline 30 & " & -10.9 & 196.0 & $\prime \prime$ & 8.2 & 0.068 & 330 \\
\hline 31 & " & -10.4 & 195.2 & $\prime \prime$ & 12.5 & 0.034 & \\
\hline 32 & 2015-08-01, 23:55 & -11.0 & 195.8 & $02: 30$ & 14.3 & 0.053 & \\
\hline 33 & " & -11.0 & 196.2 & " & 10.8 & 0.043 & 220 \\
\hline 34 & 2015-09-14, 18:47 & $-25^{*}$ & $198 *$ & $14: 30$ & - & 0.39 & 1517900 \\
\hline 35 & $2015-12-13,01: 13$ & -31.5 & 204.5 & $03: 15$ & 11.2 & 0.846 & 9835 \\
\hline
\end{tabular}

Notes. To compute the cometary local time, we considered one cometary day equal to 12.4 UTC hours and disregarded local topography. We report the VIS slope and RADF at $535 \mathrm{~nm}$ at maximum intensity. Instantaneous mass, otherwise, was calculated for all extended plume, until the photometric profile dropped to the uncertainty RADF level ( 0.005). Because we were almost unable to distinguish some neighboring dust plumes, their estimated dust masses were clustered together.

rate of larger grains also rises. Conversely, we may be observing the evaporation of icy grains as a larger cross-section of the plume is insolated.

To be able to compare plumes and features, we also derived the dust mass of the plumes. Through the RADF at $649 \mathrm{~nm}$ (F22), we estimated the filling factor $f$ per pixel, that is, the fraction of radiance scattered from an optically thin dust coma $(\tau \ll 1)$, to then obtain a quantity of instantaneous dust mass per pixel. The dust filling factor is expressed as (Fink \& Rubin 2012; Fink \& Rinaldi 2015)

$f=\frac{R A D F}{w_{\lambda}\left(p_{(g)} / p_{(0)}\right)}$

where $w_{\lambda}$ is the single-scattering albedo from Fornasier et al. (2015), estimated from Hapke modeling of the surface scattering curve, and $p_{(g)}$ is the particle phase function from Bertini et al. (2017). Bockelée-Morvan et al. (2017) showed that outbursts may have significant contributions from small and high-albedo grains, thus our filling factor estimates are to be considered an upper limit. Assuming a differential grain size distribution described by $n(a) \mathrm{d} a \sim a^{h} \mathrm{~d} a$, spherical grains and bulk density of $\rho=795 \mathrm{~kg} \mathrm{~m}^{-3}$ (Fulle et al. 2016), we obtain the dust mass per pixel cross-section (Hughes 1972),

$M_{\text {pixel }}=\rho \cdot f \cdot A_{(\Delta)} \frac{\int \frac{4 \pi}{3} n_{(r)} a^{3} \mathrm{~d} a}{\int n_{(r)} \pi a^{2} \mathrm{~d} a}$,

where $A_{(\Delta)}$ is the pixel cross-section in meters as a function of the $\mathrm{S} / \mathrm{C}$ distance $\Delta$ and $a$ is the grain radius. Since the power-law index $h$ is unknown for all ejections here observed, we referred to Agarwal et al. (2017), whereas for a particular outburst a multiinstrumental study was performed to estimate $h$ from $10 \mu \mathrm{m}$ to $1 \mathrm{~mm}$ in size range. Therefore, from 10 to $150 \mu \mathrm{m}$ we integrated Eq. (4) with $h=-2.54$, from 150 to $500 \mu \mathrm{m} h=-3.0$ and $500 \mu \mathrm{m}$ to $1 \mathrm{~mm} h=-6.9$ to finally obtain the dust mass. Taking the same area that to the ejections and displacing it to the 
(a)

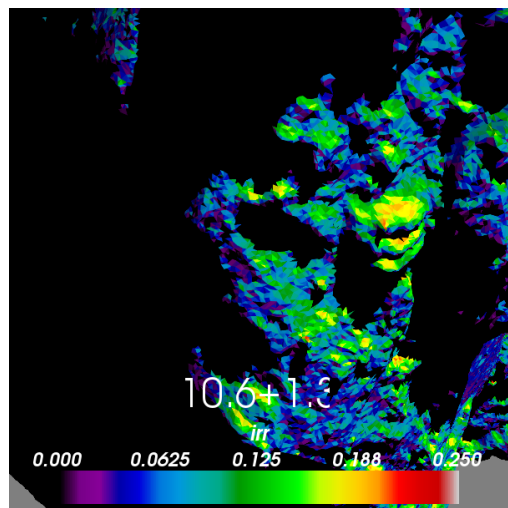

(b)



(c)



Fig. 7. Irradiance and illumination conditions over the High bank on January 16, 2015. The figures were simulated with OASIS (Jorda et al. 2010) at steps of 15 UTC min from 00:00:00 to 1:30:00 using the decimated DLR SPG SHA7 model of 500 thousand facets. Irradiance is shown in spectral color scaling alongside the cometary time at subsolar longitude plus the respective time zone of the Khonsu bank $(+1.3$ cometary hours at $\varphi=-22.5^{\circ}, \lambda=199^{\circ}$ ). (a) 2015-01-16 00:00:00. (b) 2015-01-16 00:30:00. (c) 2015-01-16 01:30:00.

quiescent inner coma, off nucleus, we are able to estimate a maximum mass overestimation of $20 \%$. We recall that the reported dust masses are only valid for radiation scattered in the dust environment $(\tau \ll 1)$ and not for illuminated surfaces $(\tau \approx 1)$. The masses we report here do not reflect the total mass ejected integrated through time, but only the actual plume framed by OSIRIS NAC.

In addition to the three massive outbursts (numbers 1,2 , and 3; 20 and 22; 34; and 35), most plumes have masses $<500 \mathrm{~kg}$. Particularly plumes $0,12,15$, and 19 compose a single steady dust-production event arising from the same site. The plume is activated after sunset and lies apparently perpendicular to the deposit (Fig. 8a).
Two bright outbursts are observed to originate from the equatorial reach of the High bank (numbers 1, and 20 and 22) instead of a single outburst, as reported by Vincent et al. (2016b). Both occurred during cometary night, 1:07 $\mathrm{h}_{\text {comet }}$ and 22:3000:30 $\mathrm{h}_{\text {comet }}$, respectively, almost one rotation apart (Fig. 8a). The first outburst is the second brightest of our collection, peaking at $R A D F_{\max }=0.6 \%\left(\alpha=89^{\circ}\right)$, with a pixel-integrated mass of $\sim 50$ tons and a very broad shape. One hour before no activity was noticed, but after $0.5-2.5 \mathrm{~h}$ later, a dust plume of $1-3$ tons remains. In the second outburst (numbers 20 and 22), we observe a packed but less massive plume ( $\sim 5$ tons) for a duration of $30 \mathrm{~min}$. One hour later, a dust plume of $800 \mathrm{~kg}$ (numbers 24 and 25 ) is still visible, but is partially dispersed after UTC 23:25, when the pixel-integrated mass drops to less than $300 \mathrm{~kg}$ (numbers 29, 30, 31, 32, and 33). All these events add up a total of 67 tons of released dust.

The September 14, 2015, outburst has been studied in detail by Bockelée-Morvan et al. (2017). Rinaldi et al. (2018), studying the same outburst with VIRTIS-M, have measured a particle velocity as high as $25.3 \pm 1.6 \mathrm{~m} \mathrm{~s}^{-1}$ for grains ranging from $0.3-5 \mu \mathrm{m}$. Here we report a mass of $1.52 \times 10^{3}$ tons of a plume extending for $8 \mathrm{~km}$ from the nucleus surface (see Fig. 3 in Vincent et al. 2016b) observed at grazing angle. According to the velocity derived by Rinaldi et al. (2018), the outburst started $5 \mathrm{~m} 20$ s before the OSIRIS NAC observation. This is by far the most massive outburst that was observed to arise from the Khonsu bank. The outburst was triggered during the afternoon and presents a complex shape with two components in V-shape. No NAC or WAC color sequences were obtained for this event.

On December 13, 2015, at 1:13 h (Fig. 8b), a very bright collimated outburst was observed at $100 \mathrm{~km}$ from the nucleus. We estimated its integrated mass at $\sim 10$ tons. The outburst took place at 3:15 $\mathrm{h}_{\text {comet }}$ with sunrise occurring $2 \mathrm{~h}_{\text {comet }}$ later. One hour later, the dust plume became fainter, and its radiance and colors were convolved with the recently illuminated surface.

\section{Discussion}

\subsection{Sources of local activity}

In Sect. 4 we described the morphological changes in the Khonsu bank during the course of $67 \mathrm{P}$ through perihelion. In the preceding Sect. 6, we measured the properties and coordinates of all dust plumes that emanated during August 1, September 14, and December 13, 2015. To associate the surface changes with activity, we overplotted all source coordinates in Fig. 9 on the frame of January 28, UTC 01:48. This is a particular frame where the bank was well insolated. In what follows, we discuss the nature of every source's site alongside the properties of their associated activity.

\subsubsection{Low bank: Patch 1 and surroundings}

The icy Patch 1 and its surroundings are associated with four faint narrow plumes (numbers 21, 26, 27, and 28) with a total mass of $\sim 650 \mathrm{~kg}$. Observed activity is trigged after insolation ceased for $\sim 4 h_{\text {comet }}$. The Low bank is partially visible on August 1, 2015, at 22:25 and 22:55, but three of four faint plumes are only observed at 22:55. Previous studies (Oklay et al. 2016b; Knollenberg et al. 2016; Pajola et al. 2017; Agarwal et al. 2017) have demonstrated the link between ice-enriched zones and outbursts. However, we observe above Patch 1 what better corresponds to dust elevation carried by delayed sublimation of the ice-rich terrain that lasts longer into local night. Activity 

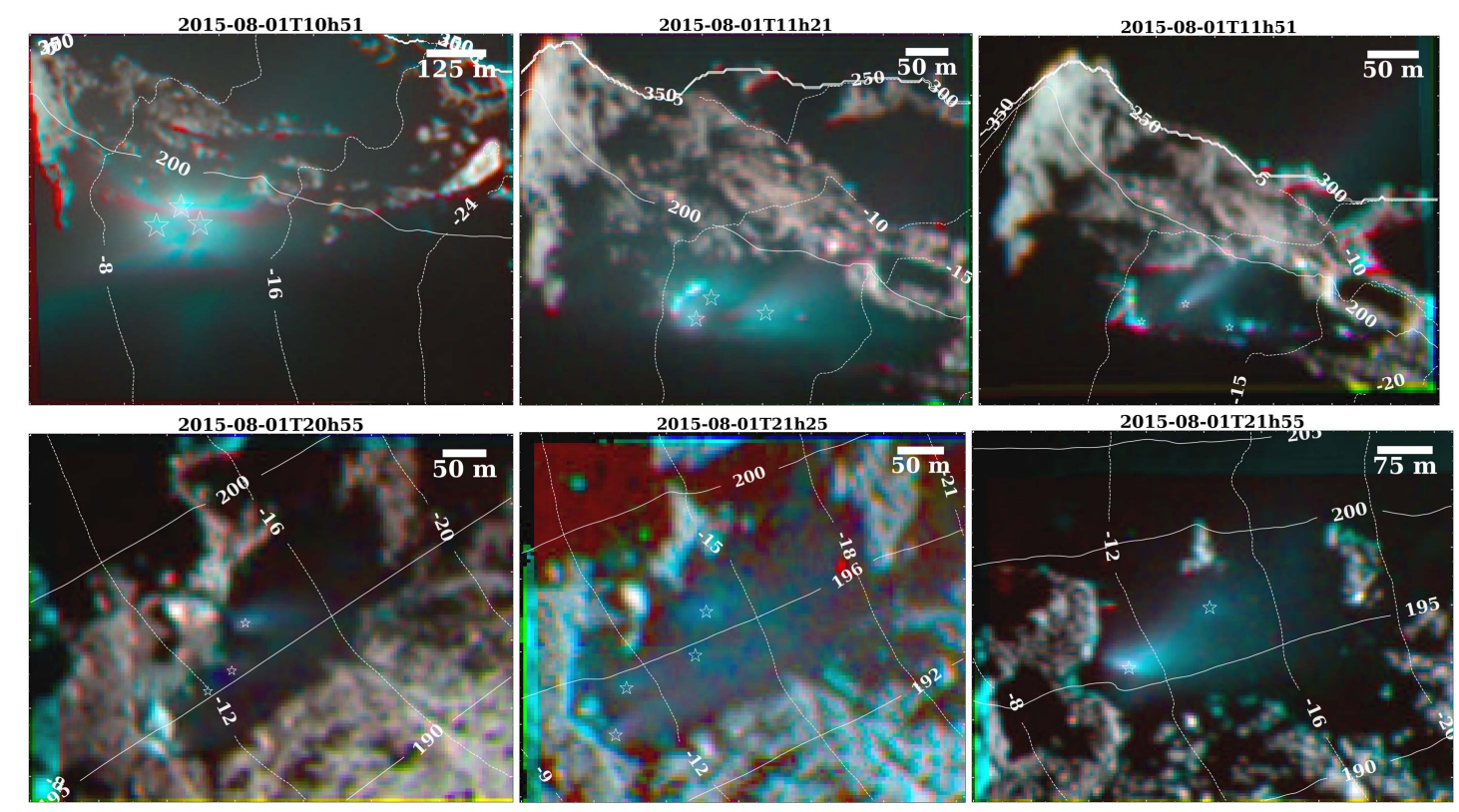

(a)

2015-12-13T01h13


Fig. 8. Plethora of transient events emanating from the Khonsu bank. False-color RGB (F41-F23-F24) composites of some mass ejections captured by OSIRIS NAC. The locus of maximum intensity is marked by a white star. (a) some of the color sequences on August 1, 2015, that show activity emanating from the Khonsu bank. The first and last frames (UTC 10:51 and 21:55) show the two outbursts that arise from the same site. In the aftermath of the first outburst (UTC 11:21 and 11:51), we observe a collimated morning event emanating from nearby. In the frames of UTC 20:55 and 21:25, just before sunset, jumping boulder 2 and deformation are particularly visible. $(b)$ the outburst of December 13, 2015, and its source in the southern outskirts of the Low bank, near the pancake feature.

near sunset has also been observed in other sites (Shi et al. 2016, 2018). It shows that subsurface heat diffusion and thermal-lagged water sublimation (Prialnik et al. 2008) at $1.8 \mathrm{AU}$ are enough to sustain activity for several hours after solar input has ceased.

Similar to Shi et al. (2016), we narrowed the thermal skin depth $Z$ based on the timescale $\tau_{k}$ of thermal conduction of the activity (Huebner et al. 2006). From the equation $Z=\sqrt{\tau_{k} \kappa / \pi \rho c}$, where we assumed a heat capacity $c=1000 \mathrm{~K}^{-1} \mathrm{~kg}^{-1}$ and an effective conductivity $\kappa=0.005 \mathrm{~W} \mathrm{~K}^{-1} \mathrm{~m}^{-1}$, we derived
$Z \approx 5 \mathrm{~mm}$ based on $\tau_{k}=2 h$. This value is encompassed by the average diurnal skin depth of $8.5 \mathrm{~mm}$, based on half a rotation period of $67 \mathrm{P}$, and it is also compatible with the $1 \mathrm{~mm}$ layer of global water ice depletion found by VIRTIS-M (Capaccioni et al. 2015). Therefore, the lasting blue spectral slope of Patch 1 indicates a gentle ice replenishment mechanism and/or continuous dust removal that has been ongoing on the top few millimeters, which is probably the reason that no pronounced surface change is visible in the images. 




Fig. 9. All ejection sources projected onto the NAC image of January 28, 2016, UTC 01:48 (F41-F23-F24). Position for event 33 (-25 ${ }^{\circ}, 198^{\circ}$, September 14, UTC 18:47) of Vincent et al. (2016b) is represented by a white star, while the white dotted ellipse shows the reprojected spatial uncertainty on the image. Most of the documented events cluster in the equatorial outskirts of the High Khonsu bank, with sparse sources located in the Low Khonsu bank. The size of the red markers is proportional to the median uncertainty of all sources.

\subsubsection{Low bank: thick dust layer}

The source location of the September 14, 2015, 18:47 (\#34) outburst presents the largest spatial uncertainty because of the grazing angle that has been observed (Vincent et al. 2016b), encompassing most of the Low bank as a result (the white dotted ellipse in Fig. 9). The magnitude and central position of this outburst matches the formation of cavity $\mathrm{C} 6$, that is, a vanishing mass of $\sim 2.6 \times 10^{7} \mathrm{~kg}$.

The ejected dust mass corresponds to a maximum of $\sim 6 \%$ of the total vanished mass, equivalent to a lost stratum of $\sim 65 \mathrm{~cm}$. Stretching the lower limit of grain-size distribution to $0.1 \mu \mathrm{m}$ or raising the grain-size distribution of large grains to -3.5 only increases the dust mass to $\sim 10 \%$.

A possible way to access this conundrum is to equate the cavity mass and the ejected dust mass. This assumption is only valid if we consider that the observed dust mass is the total dust mass that has been ejected in a single short production (Knollenberg et al. 2016). Free parameters are the dust-to-ice grain ratio $\xi$ and the released gas mass $M_{\mathrm{gas}}$, both related to the volatile content in the reservoir. $\mathrm{C} 1$ and $\mathrm{C} 2$ have indicated that ice pockets a few meters wide from each other can be found in the nucleus. Furthermore, Bockelée-Morvan et al. (2017) measured a high bolometric albedo and high superheat factor with the VIRTIS-H instrument, indicating an important contribution of ice/silicate submillimeter grains in the ejecta. When we consider that the outburst is composed of dark carbon-organic materials and ices, the conservation of mass yields (based on Eqs. (4) and (5))

$$
M_{T}=\frac{(1+\xi) \cdot M_{O} \cdot w_{D}}{w_{I}+\xi w_{D}}+M_{\mathrm{gas}}
$$

where $M_{T}$ and $M_{O}$ are the masses of the cavity (C6, Table 3) and the outburst mass of event 34 (Table 4). $w_{I} \approx 0.99$ and $w_{D}=0.047$ are the single-scattering albedos of pure ice and dark carbon-organic dust at $649 \mathrm{~nm}$, respectively. In the equation, we assume an areal mixture of two granular components of the albedo. In Fig. 10 we hence present the gas-to-outburst mass ratio as a function of dust-to-ice grain ratio. For $67 \mathrm{P}$, the average dustto-ice ratio has a nominal value of 6-7.5 (Fulle et al. 2016, 2017). Assuming that $\mathrm{C} 6$ has a dust-to-ice grain ratio $<6$, we must have $98-99 \%$ (gas-to-outburst mass $\approx 50-250$ ) of the mass of C6 that is volatilized. The gas-to-outburst mass may be narrowed down to $\sim 60-170$ when a silicate component of 0.2 is introduced into $w_{D}$. The lower limit is comparable to that found by Grün et al. (2016) for the February 16, 2016, outburst $(\approx 40)$. In conclusion, most of the cavity mass would be contained in $M_{\text {gas }}$, with refractories and silicates composing only a few percent. This could indicate to very ice-rich sub-surface pockets underneath that are almost completely volatilized during such events.

Conversely, another hypothesis is that these brighter events are restricted and that most of the mass is depleted through a mild and steady mass removal. During four months of major cometary activity, a very low steady production of $\sim 3 \mathrm{~kg} \mathrm{~s}^{-1}$ 


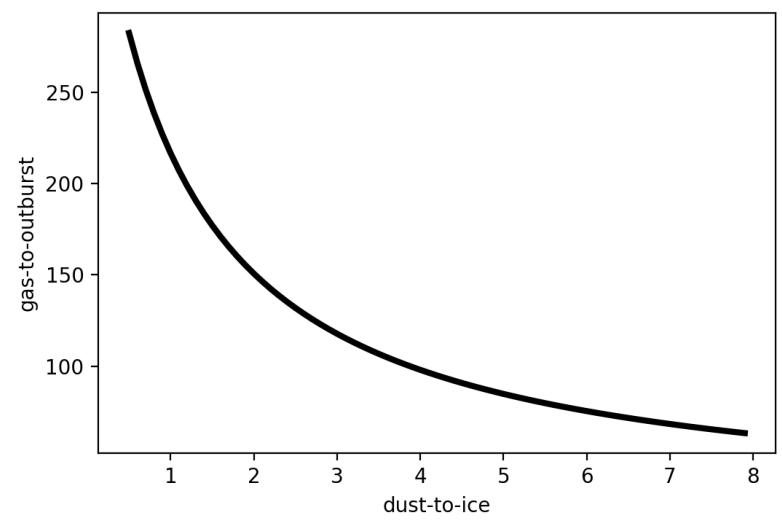

Fig. 10. Gas-to-outburst mass ratio vs. dust-to-ice grain ratio constrained based on the masses of C6 and event 35. We consider that the observed dust mass is the total dust mass that has been ejected in a single short production. The relation calculated from Eq. (6) in the interval of dust-to-ice is 0.5 to 8 .

$\left(6.5 \times 10^{-4} \mathrm{~kg} \mathrm{~s}^{-1} \mathrm{~m}^{-2}\right)$ would be enough to deplete the mass of C6.

\subsubsection{Low bank: prompter of jumping boulder 1}

After the perihelion passage, El-Maarry et al. (2017) noted the rolling of jumping boulder 1 (F2) from a higher ground in the rough terrain situated south from the deposit. El-Maarry et al. (2017) raised the hypothesis that a collimated jet may have lifted or pushed jumping boulder 1 downhill from its original position. A minimum gas production rate of $8.5 \times 10^{23} \mathrm{~m}^{-2} \mathrm{~s}^{-1}$ was estimated (El-Maarry et al. 2017). Another hypothesis relates to local seismic vibrations caused by a neighboring active source.

The original position of F2 is only $100 \mathrm{~m}$ east of the source of the massive outburst ( $\sim 10$ tons) on December 13, 2015, UTC 01:13 (event 35). Its source is particularly visible one hour later, showing an $18-\mathrm{m}$ Patch that is 1.7 times brighter $\left(R A D F_{\max }=1.3 \%\right)$ and $50 \%$ bluer $(8-9 \% / 100 \mathrm{~nm})$ than the surroundings at $\alpha=88^{\circ}$. This Patch was not observed in high resolution, but was present in March 2015 (Fig. 3a). This event showed a similar behavior as the July 3, 2016, outburst (Agarwal et al. 2017), a very massive morning jet arising from an ice-rich area a few meters wide that is concealed by an outcrop.

Finally, the actual observation of this massive outburst indicates that the source has not been exhausted, since F2 had already rolled when this event took place. We estimate that a previous outburst of similar or higher magnitude that perturbed F2 probably took place between the middle of August and the end of October 2015.

\subsubsection{High bank: retreating wall}

The retreating wall $(\mathrm{F} 1)$ is associated with events $0,12,13,14$, 15,16 , and 19 . These persistent plumes are activated or remained active after sunset and present a mass of $\sim 500-700 \mathrm{~kg}$. A low steady mass loss of $7 \mathrm{~kg} \mathrm{~s}^{-1}\left(8.3 \times 10^{-4} \mathrm{~kg} \mathrm{~s}^{-1} \mathrm{~m}^{-2}\right)$ would be required during three months to deplete it. This low loss rate may explain why no associated bright outburst was detected.

The morphological phenomena of the retreating wall are reminiscent of the retreating smooth terrains observed in 9P/Tempel 1 (Thomas et al. 2013; Veverka et al. 2013), where $\sim 50 \mathrm{~m}$ of length was lost. At different size scales, retreating discontinuities have also been observed in smooth terrains on Imhotep (Groussin et al. 2015) and Ma'at (Hu et al. 2017).

\subsubsection{High bank: jumping boulder 2}

About half of the activity events (18/36, numbers 1, 2, 3, 4, 5, 8, $9,11,17,18,20,22,24,29,30,31,32$, and 33) has emanated from the jumping boulder 2 (F3) site, and all occurred on August 1, 2015 (Fig. 9). Because of the noticeable deformation (F5) on the equatorial cliff $\left(-10^{\circ}\right.$ latitude), we can narrow its apparition down to between UTC 08:00 (Fig. 5b) and 20:55 (Fig. 8a) on the same date, thus linking it to the activity emanating from the High bank in the same time frame. Remarkably, at UTC 20:55, F3 is bluer $(8.7-10.4 \% / 100 \mathrm{~nm}$ against an average VIS slope of $18 \% / 100 \mathrm{~nm}$ ), indicating that F3 has partially lost its surface dust cover shortly after the outburst. Similar scratches of ice-enrichment resting on boulders have been noted before by Deshapriya et al. (2018).

The outburst event 1, 2, and 3 of August 1, 2015, UTC 10:51 might have been observed only a few minutes after the reshaping of the equatorial cliff in the High bank. Subsequently, plumes 4, $5,8,9$, and 11 (3-2 tons) are an afterproduct of event 1, 2, and 3, which originally carried a dust mass of $\sim 50$ tons (Sect. 6). One rotation later, we then observed a second outburst (number 22), and ongoing activity before and after this event. The process continued until the surface was depleted in volatiles and adopted the average spectral slope seen in 2016 (Fig. 5c).

In the scenario of cliff thermal erosion, where freshly exposed volatile-rich scarps subsequently drive outbursts and activity (Vincent et al. 2016a,b; Pajola et al. 2017), the 50-mwide boulder would simply be a consolidated remnant from the equatorial cliff collapse. In this case, the outburst would not have had a high enough gas production rate to move $\mathrm{F} 3$ from its original position. This scenario cannot be invalidated by our observations, but there is one circumstantial weakness to this scenario that is worth addressing: the lack of any noticeable talus under the deformed equatorial cliff. Mass wastes formed under cliffs have shown boulder-size distribution power indexes ranging from -2.5 to -4 for $1.5-15 \mathrm{~m}$ in diameter (Vincent et al. 2016a; Pajola et al. 2017). Therefore, the lack of blocks for diameters between 7 and $50 \mathrm{~m}$ is unusual, whereas $7 \mathrm{~m}$ is the largest boulder after F3 that we can detected under the deformation in the June 25, 2016, UTC 06:09 F22 image. Furthermore, 50 -m-wide boulders have been never noted in a talus by previous boulder-size distribution analyses (Vincent et al. 2016a; Pajola et al. 2017).

An alternative scenario is that $\mathrm{F} 3$ fell again into Khonsu bank and caused outburst 1 . Lifting a 50 -m-wide boulder requires a gas production rate of $1.4 \times 10^{24} \mathrm{~m}^{-2} \mathrm{~s}^{-1}\left(R_{\mathrm{eff}}=25 \mathrm{~m}\right.$, Eq. (3)), which is roughly similar to the theoretical production rate achieved by the July 3, 2016, outburst if it is carried by an amorphous-to-crystalline transition $\left(1.1 \times 10^{24} \mathrm{~m}^{-2} \mathrm{~s}^{-1}\right.$, Agarwal et al. 2017). Thus, F3 could have been accelerated to near escape velocity $\left(\sim 0.8 \mathrm{~m} \mathrm{~s}^{-1}\right)$ by a past energetic outburstat the end of July 2015, such as the jet of July 29, 2015, UT 13:34 (Lin et al. 2017) or another unobserved event. F5 has no distinguishable crater shape, however, likely because the boulder was at low velocity and maybe first touched the tip of the cliff during the re-fall, thus deforming but attenuating the final surface shock. F3 has not been clearly shattered, indicating significant internal material consolidation. Therefore, the lack of talus could be explained by the grinding process, product of the shock, that could have reduced the cliff material to centimeter-to-millimetersized grains, a state in which unconsolidated material is more easily lifted by out-gassing. This scenario is partially corroborated by the detection of meter-sized objects floating around the nucleus during perihelion approach that were observed to 
be as fast as $180 \mathrm{~km}$ (Fulle et al. 2016); and other "boulder displacement" occurrences (El-Maarry et al. 2017).

Subsequently, plumes $4,5,8,9$, and 11 (3-2 tons) are an after-product of event 1,2 , and 3, which originally carried a dust mass of $\sim 50$ tons (Sect. 6). One rotation later, we then observed a second outburst (number 22) and ongoing activity before and after this event. Activity is now conducted by sublimation of exposed ices by the low-velocity impact and thermal interplay with the local topography. The process continued until the surface was depleted and adopted the average spectral slope seen in 2016 (Fig. 5c).

\subsection{Total lost mass}

The sum of masses of all morphological changes that took place in the bank can give an estimate of its contribution to the total ejected mass by the nucleus. The morphological units $\mathrm{C} 1, \mathrm{C} 2$, and $\mathrm{C} 3$ and mound F4 are in the same order of mass as the cliff collapse observed by Pajola et al. $\left(2017 ; 1.1 \times 10^{5} \mathrm{~kg}\right)$. However, if we account for all appearing cavities, the retreating wall (F1) and the deformation/excavation (F5), the Khonsu bank is responsible for $1.7 \times 10^{8} \mathrm{~kg}$, that is, $1.5-4.2 \%$ of the total released mass according to Paetzold et al. $\left(2017 ; 1.1 \pm 0.3 \times 10^{10} \mathrm{~kg}\right)$ or Thomas et al. $\left(2015 ; 4 \times 10^{9} \mathrm{~kg}\right.$ ), assuming a dust-to-ice ratio of 7.5 (Fulle et al. 2017). Cavities only come out with $2.8 \times 10^{7} \mathrm{~kg}$, or $\sim 1 \%$. This indicates that as much as $\sim 25$ similar active zones are sufficient to sustain the entire mass loss. The dust plumes otherwise only contribute with $1.6 \times 10^{6} \mathrm{~kg}, \sim 1 \%$ of the mass of vanished features. This conundrum is discussed in Sect. 7.1.2.

\subsection{Activity mechanism}

Mass ejections and morphological changes observed in the particular Khonsu bank paint the variety of mechanisms in broad strokes that trigger outbursts on the surface of 67P. Recently, erosion of cliffs, caused by receding fractures caused by diurnal and seasonal heating that leads to wall weakening and their final collapse, has been proposed (Vincent et al. 2016a,b) to explain some parts of the link between outbursts and comet surface morphology. The mechanism is corroborated by the observation of mass wastes close to cliffs and by the detection of an actual cliff collapse (Pajola et al. 2017).

Cliff erosion is considered to be gentler than the longer standing hypothesis invoking build-up of devolatilized gas pockets. Thermal waves penetrating ice-rich sub-surface layers and reaching a critical pressure would overcome the tensile strength of the top-dust layer and be released as a strong collimated jet or escape to space through percolation (Belton et al. 2008). The model for the formation of sinkholes proposed by Vincent et al. (2015) is very similar to this hypothesis, with the difference that the gas pocket forms a subsurface cavity that ultimately collapses because the ceiling above weakens.

Alternatively, Agarwal et al. (2017) proposed the short-time transition from amorphous-to-crystalline water ice to explain a massive outburst (up to 139 tons) that arose from Imhotep. Water vapor that recondensates under low pressure and temperatures below $160 \mathrm{~K}$ assumes an amorphous configuration. When heated up to $>180 \mathrm{~K}$, the ices are exothermically transformed into hexagonal structure in matters of minutes or days. The latent heat of the transition is about $9 \times 10^{4} \mathrm{~J} \mathrm{~kg}^{-1}$ (González et al. 2008), which means that this process increases local temperatures up to $\sim 240 \mathrm{~K}$ and is sustained very near to the surface. This mechanism may give rise to very localized phenomena and strongly depends on periodic shadowing, thus local topography.
In our observations of the Khonsu bank, we captured intense changes (Tables 3 and 2), excavations of 1.3-14 m deep, and cavities forming meters from cliffs, but none seems related to cliff collapse as observed in image sequences by Pajola et al. (2017). Striking evidence of sub-surface heterogeneity comes from the comparison of the VIS spectral slopes of cavities $\mathrm{C} 1$ and $\mathrm{C} 2$ : while $\mathrm{C} 2$ shows zones of ice-enriched smooth terrains, $\mathrm{C} 1$ has the same average redder VIS spectrum as the nucleus. Both cavities, formed sometime between March and December 2015, are only $30 \mathrm{~m}$ apart and aligned to the longitude of $196^{\circ}$. They experience the same amount of solar irradiance and shadowing from eastern cliffs and the enclosing $700 \mathrm{~m}$ high Apis plateau. They exhibit the same characteristic depth of $\sim 3-5 \mathrm{~m}$, but each sustains a different VIS spectrum (Fig. 6b).

Other evidence of sub-surface heterogeneity also comes from the two features C6 and F1. After perihelion, VIS spectra indicate depletion and no sign of ices on their surfaces. The internal ices of both features were lost through the same gas release that drove their mass loss. However, they are no farther than $50 \mathrm{~m}$ from icy patches 1 and 3 .

Therefore, we observed plenty of circumstantial evidence of sustained and very localized pockets of ice underneath. However, could they also help us narrow down the driving mechanism behind activity? The hypothesis of Belton et al. (2008) demands centimeters to meters of sintered or frozen surface to allow the gas reservoir to build up, only to be released through cracks formed through ongoing thermal stress. The surface aspect of a mechanism described in Agarwal et al. (2017) is otherwise not clear; as percolation is needed, it is conceivable that a very smooth and porous uppermost layer is to be expected and not a cracked or eroded surface.

Morphologically, the Khonsu bank preserves its general smooth dusty texture before and after perihelion without any sign of cracks or steep boulder-size distribution up to a few decimeters. The base of patches is very smooth to a scale of $150 \mathrm{~cm}$. Their sustained blue color in daylight and throughout several months is very different from a surface that is expected to build up gas underneath. The VIS spectral slope shows us that before perihelion, the poorly illuminated high bank has a cyclic formation of frost from gas that percolated and diffused onto the surface. After perihelion, almost all enriched surfaces are concealed by cliffs, where the recondensation cycle can still be repeated.

On the other hand, the boulder-size frequency distribution in the environment of Patch 1 indicates a sintered crust underneath. This is corroborated by measurements from MUPUS/Philae (Biele et al. 2015). The observation of large blocks in the surroundings, rather than in the base of Patch 1 , indicates an energetic past event that expelled and scattered these boulders. The evidence, in this case, better complies with the hypothesis of sub-surface gas storage.

We have also witnessed energetic events such as the possible origin of jumping boulder 2 and the September 14, 2015, and December 13, 2015, outbursts. However, it is not clear if the event of September 14, 2015, completely formed C6. Both C6 and F1 could have been depleted through a low steady dust production rate. The diurnal cycle alongside a low thermal inertia leads to a time-lagged thermal-wave penetration of a few millimeters and can sustain outgassing and dust removal through hours after sunset (Shi et al. 2016, 2018). During the cooling down late at night, the uppermost layer is again drenched by ices that percolate and recondensate from ice-rich layers below. This cycle is reignited again and again during the southern summer, sustaining the dust production for several months. 
Finally, the outburst of December 13, 2015, denotes a more complex mechanism. This event is at least the second to emanate from the same source, a blue bright patch near the Low bank. Since the source was not exhausted, more than one outcrop collapse or a near-surface energy build-up likely drive the activity there.

In conclusion, we noted that the morphological changes operating in the Khonsu bank are very diverse. Their associated activity also ranges wide in magnitude and daytime. We did not observe any systematic occurrence in the mass ejections or in their sources that could indicate the predominance of a specific mechanism, even in a very small area such as the Khonsu bank.

\section{Summary and conclusion}

The OSIRIS/Rosetta observations of the southern Khonsu region have shown that an apparently smooth terrain of about $100 \times 700 \mathrm{~m}^{2}$ has been highly active during the perihelion approach in the second half of 2015 (Deshapriya et al. 2016, 2018; Laeuter et al. 2019). Observed and unobserved activity has reshaped the zone, and several morphological changes were witnessed through image color sequences obtained during 2016. We then estimated the masses and dimensions of the morphological features, and also the dust mass of their corresponding activities and outbursts using grain-size distribution and grain density reported in the literature (Fulle et al. 2016; Agarwal et al. 2017). We can summarize our findings as follows:

- Over a period of one year and a half, we saw the formation of four cavities in the surface of the Khonsu bank with depths ranging from 1.3 to $14 \mathrm{~m}$. One cavity appeared where a $15-\mathrm{m}$-high mound was located, three harbor iceenriched surfaces, and the largest $(105 \times 42 \mathrm{~m}$ in length and $10-14 \mathrm{~m}$ deep) is the most plausible source of the September 14, 2015, outburst (Vincent et al. 2016b; Bockelée-Morvan et al. 2017). Circumstantial evidence through spectrophotometric analysis and also source/ outburst mass comparison likely indicates the presence of ice-rich pockets at tens of meters underneath.

- A scarp has retracted near the equator between August 1, 2015, and October 31, 2015, an equivalent mass of $(5.3 \pm 0.4) \times 10^{7} \mathrm{~kg}$. Still, no associated outburst was detected, leading us to estimate a low steady mass loss of $7 \mathrm{~kg} \mathrm{~s}^{-1}$ to completely deplete this feature.

- The appearance of a 50-m-wide boulder and an associated deformation/excavation to the equatorial cliff is connected to the outburst of August 1, 2015, UTC 10:51 (dust mass of $\sim 50$ tons). We evoked two scenarios to explain this appearance: a larger remnant of a thermal cliff erosion and collapse (Vincent et al. 2016a; Pajola et al. 2017), or gas lifting after some bright outburst at the end of July 2015 that then fell back into the Khonsu bank. It is worth further investigation whether this configuration is unique or if other similar large boulders linked to surface deformations/excavations were observed in other parts of the nucleus. Particularities in the deformation (whether near cliffs, cavities, or craters) may also reveal which scenario is more likely.

- Boulder-size frequency distribution of the wasting surrounding the ice-enriched Patch 1 exhibits a power-law index of $-1.6 \pm 0.01$ for 1.2 to $10 \mathrm{~m}$ range size, a flat slope when compared to -3.6 , for the entire nucleus (Pajola et al. 2015) and to -3.1 for all of Khonsu (Deshapriya et al. 2016). As Patch 1 is situated in a cavity that is roughly $3 \mathrm{~m}$ deep, this may indicate a sintered crust at the equivalent depth. The lack of large blocks at the base of the patch indicates a formation by thermal cracking, followed by a release of sub-surface stored energy that scattered the fragments off. Patch 1 is also associated with four emanating dust plumes two cometary hours after sunset.

- The Khonsu bank is responsible for $1.7 \times 10^{8} \mathrm{~kg}$ of released mass estimated from morphological changes, that is, $1.5-4.2 \%$ of the total mass loss experienced by $67 \mathrm{P}$, according to recent estimations (Thomas et al. 2015; Paetzold et al. 2017). This indicates that as much as 25 similar active zones are sufficient to sustain the entire mass loss.

Acknowledgements. P.H.H. would like to thank Alice Bernard for her support. This work was funded by the DIM-ACAV project (Île-de-France). OSIRIS was built by a consortium of the Max-Planck-Institut für Sonnensystemforschung, Göttingen, Germany, CISAS-University of Padova, Italy, the Laboratoire d'Astrophysique de Marseille, France, the Instituto de Astrofísica de Andalucia, CSIC, Granada, Spain, the Research and Scientific Support Department of the European Space Agency, Noordwijk, The Netherlands, the Instituto Nacional de Técnica Aeroespacial, Madrid, Spain, the Universidad Politéchnica de Madrid, Spain, the Department of Physics and Astronomy of Uppsala University, Sweden, and the Institut für Datentechnik und Kommunikationsnetze der Technischen Universität Braunschweig, Germany. The support of the national funding agencies of Germany (DLR), France (CNES), Italy (ASI), Spain (MEC), Sweden (SNSB), and the ESA Technical Directorate is gratefully acknowledged We acknowledge the financial support from the France Agence Nationale de la Recherche (programme Classy, ANR-17-CE31-0004).

\section{References}

Acton, C. H. 1996, Planet. Space Sci., 44, 65

Acton, C., Bachman, N., Semenov, B., \& Wright, E. 2018, Planet. Space Sci., 150,9

Agarwal, J., Della Corte, V., Feldman, P. D., et al. 2017, MNRAS, 469, s606 Arthur, D. W. G. 1974, Icarus, 23, 116

Barucci, M. A., Filacchione, G., Fornasier, S., et al. 2016, A\&A, 595, A102

Belton, M. J. S., Feldman, P. D., A'Hearn, M. F., \& Carcich, B. 2008, Icarus, 198, 189

Bertin, E. 2012, in Astronomical Data Analysis Software and Systems XXI, eds. P. Ballester, D. Egret, \& N. P. F. Lorente, ASP Conf. Ser., 461, 263 Bertini, I., La Forgia, F., Tubiana, C., et al. 2017, MNRAS, 469, S404 Biele, J., Ulamec, S., Maibaum, M., et al. 2015, Science, 349, aaa9816 Bockelée-Morvan, D., Rinaldi, G., Erard, S., et al. 2017, MNRAS, 469, S443 Boggs, P. T., \& Rogers, J. E. 1990, Contemporary Mathematics (Rhode Island, US: AMS), 112, 186

Boulton, G. S. 1978, Sedimentology, 25, 773

Capaccioni, F., Coradini, A., Filacchione, G., et al. 2015, Science, 347, 628 Chappelow, J. E., \& Sharpton, V. L. 2002, Meteor. Planet. Sci., 37, 479 De Sanctis, M. C., Capaccioni, F., Ciarniello, M., et al. 2015, Nature, 525, 500 Deshapriya, J. D. P., Barucci, M. A., Fornasier, S., et al. 2016, MNRAS, 462, S274

Deshapriya, J. D. P., Barucci, M. A., Fornasier, S., et al. 2018, A\&A, 613, A36 El-Maarry, M. R., Thomas, N., Gracia-Berná, A., et al. 2016, A\&A, 593, A110 El-Maarry, M. R., Groussin, O., Thomas, N., et al. 2017, Science, 355, 1392 Feaga, L. M., A’Hearn, M. F., Sunshine, J. M., Groussin, O., \& Farnham, T. L. 2007, Icarus, 190, 345

Fink, U., \& Rinaldi, G. 2015, Icarus, 257, 9

Fink, U., \& Rubin, M. 2012, Icarus, 221, 721

Fornasier, S., Hasselmann, P. H., Barucci, M. A., et al. 2015, A\&A, 583, A30

Fornasier, S., Mottola, S., Keller, H. U., et al. 2016, Science, 354, 1566

Fornasier, S., Hoang, V. H., Hasselmann, P. H., et al. 2019, A\&A, 630, A7 (Rosetta 2 SI)

Fulle, M., Marzari, F., Della Corte, V., et al. 2016, ApJ, 821, 19

Fulle, M., Della Corte, V., Rotundi, A., et al. 2017, MNRAS, 469, S45

Golombek, M., \& Rapp, D. 1997, J. Geophys. Res., 102, 4117

González, M., Gutiérrez, P. J., Lara, L. M., \& Rodrigo, R. 2008, A\&A, 486, 331

Groussin, O., Sierks, H., Barbieri, C., et al. 2015, A\&A, 583, A36

Grün, E., Agarwal, J., Altobelli, N., et al. 2016, MNRAS, 462, S220

Hapke, B. 2008, Icarus, 195, 918

Hasselmann, P. H., Barucci, M. A., Fornasier, S., et al. 2016, Icarus, 267, 135

Hasselmann, P. H., Barucci, M. A., Fornasier, S., et al. 2017, MNRAS, 469, S550

Hu, X., Shi, X., Sierks, H., et al. 2017, A\&A, 604, A114

Huebner, W. F., Benkhoff, J., Capria, M.-T., et al., 2006, Heat and Gas Diffusion in Comet Nuclei (Noordwijk: ESA Publications Division)

Hughes, D. W. 1972, Planet. Space Sci., 20, 1949

Jorda, L., Spjuth, S., Keller, H. U., Lamy, P., \& Llebaria, A. 2010, Proc. SPIE, 7533, 753311 
Jorda, L., Gaskell, R., Capanna, C., et al. 2016, Icarus, 277, 257

Jost, B., Pommerol, A., Poch, O., et al. 2017, Planet. Space Sci., 145, 14

Keller, H. U., Barbieri, C., Lamy, P., et al. 2007, Space Sci. Rev., 128, 433

Keller, H. U., Mottola, S., Davidsson, B., et al. 2015, A\&A, 583, A34

Knollenberg, J., Lin, Z. Y., Hviid, S. F., et al. 2016, A\&A, 596, A89

Laeuter, M., Kramer, T., Rubin, M., \& Altwegg, K. 2019, MNRAS, 483, 852

Lai, I.-L., Ip, W.-H., Su, C.-C., et al. 2016, MNRAS, 462, S533

Lee, P., Veverka, J., Thomas, P. C., et al. 1996, Icarus, 120, 87

Lin, Z.-Y., Knollenberg, J., Vincent, J.-B., et al. 2017, MNRAS, 469, S731

Mottola, S., Lowry, S., Snodgrass, C., et al. 2014, A\&A, 569, L2

Oklay, N., Sunshine, J. M., Pajola, M., et al. 2016a, MNRAS, 462, S394

Oklay, N., Vincent, J.-B., Fornasier, S., et al. 2016b, A\&A, 586, A80

Oklay, N., Mottola, S., Vincent, J.-B., et al. 2017, MNRAS, 469, S582

Paetzold, M., Andert, T., Hahn, M., et al. 2016, Nature, 530, 63

Paetzold, M., Andert, T., Barriot, J.-P., et al. 2017, AAS/Division for Planetary Sciences Meeting, 49, 509.01

Pajola, M., Vincent, J.-B., Güttler, C., et al. 2015, A\&A, 583, A37

Pajola, M., Höfner, S., Vincent, J. B., et al. 2017, Nat. Astron., 1, 0092

Pommerol, A., Thomas, N., El-Maarry, M. R., et al. 2015, A\&A, 583, A25

Preusker, F., Scholten, F., Matz, K.-D., et al. 2015, A\&A, 583, A33

Preusker, F., Scholten, F., Matz, K.-D., et al. 2017, A\&A, 607, L1

Prialnik, D., A'Hearn, M. F., \& Meech, K. J. 2008, MNRAS, 388, L20

Raponi, A., Ciarniello, M., Capaccioni, F., et al. 2016, MNRAS, 462, S476

Rinaldi, G., Fink, U., Doose, L., et al. 2016, MNRAS, 462, S547

Rinaldi, G., Bockelée-Morvan, D., Ciarniello, M., et al. 2018, MNRAS, 481, 1235

Rublee, E., Rabaud, V., Konolige, K., \& Bradski, G. 2011, in Proceedings of the International Conference on Computer Vision (IEEE Computer Society) 2564

Shi, X., Hu, X., Sierks, H., et al. 2016, A\&A, 586, A7

Shi, X., Hu, X., Mottola, S., et al. 2018, Nat. Astron., 2, 562

Shkuratov, Y., Kaydash, V., Korokhin, V., et al. 2011, Planet. Space Sci., 59, 1326

Spohn, T., Knollenberg, J., Ball, A. J., et al. 2015, Science, 349, aaa9816

Thomas, P., A'Hearn, M., Belton, M. J. S., et al. 2013, Icarus, 222, 453

Thomas, N., Davidsson, B., El-Maarry, M. R., et al. 2015, A\&A, 583, A17

Tubiana, C., Güttler, C., Kovacs, G., et al. 2015, A\&A, 583, A46

van der Walt, , S., Schonberger, J. L., Nunez-Iglesias, J., et al. 2014, PeerJ 2, e453

Veverka, J., Klaasen, K., A’Hearn, M., et al. 2013, Icarus, 222, 424

Vincent, J.-B., Bodewits, D., Besse, S., et al. 2015, Nature, 523, 63

Vincent, J.-B., Oklay, N., Pajola, M., et al. 2016a, A\&A, 587, A14

Vincent, J.-B., A'Hearn, M. F., Lin, Z.-Y., et al. 2016b, MNRAS, 462, S184

Zhu, C., Byrd, R. H., Lu, P., \& Nocedal, J. 1997, ACM Trans. Math. Softw., 23, 550

1 LESIA, Observatoire de Paris, PSL Research University, CNRS, Université Paris Diderot, Sorbonne Paris Cité, Sorbonne Universités, 5 place J. Janssen, 92195 Meudon Principal Cedex, France e-mail: hasselmann@on.br
2 Department of Astronomy, University of Maryland College Park, MD 20742-2421, USA

3 Center for Technical Physics, Institute of Physics, Vietnam Academy of Science and Technology, Hanoi, Vietnam

${ }^{4}$ Max-Planck-Institut für Sonnensystemforschung, Justus-vonLiebig-Weg, 3, 37077 Göttingen, Germany

5 Department of Physics and Astronomy "Galileo Galilei", University of Padova, Via Marzolo 8, 35131 Padova, Italy

${ }^{6}$ Center of Studies and Activities for Space (CISAS) "G. Colombo", University of Padova, Via Venezia 15, 35131 Padova, Italy

7 CNR-IFN UOS Padova LUXOR, Via Trasea, 7, 35131 Padova, Italy

${ }^{8}$ Laboratoire d'Astrophysique de Marseille, UMR 7326 CNRS \& Université Aix-Marseille, 38 rue Frédéric Joliot-Curie, 13388 Marseille Cedex 13, France

9 Centro de Astrobiologia, CSIC-INTA, 28850 Torrejon de Ardoz, Madrid, Spain

${ }^{10}$ International Space Science Institute, Hallerstrasse 6, 3012 Bern, Switzerland

11 Scientific Support Office, European Space Research and Technology Centre/ESA, Keplerlaan 1, Postbus 299, 2201 AZ Noordwijk ZH, The Netherlands

12 Jet Propulsion Laboratory, M/S 183-401, 4800 Oak Grove Drive, Pasadena, CA 91109, USA

13 Physics Department, Auburn University, 206 Allison Laboratory, Auburn, AL 36849, USA

14 INAF, Osservatorio Astronomico di Padova, Vicolo dell'Osservatorio 5, 35122 Padova, Italy

15 Department of Industrial Engineering, University of Padova, Via Venezia 1, 35131 Padova, Italy

${ }^{16}$ Faculty of Engineering, University of Trento, Via Mesiano 77, 38121 Trento, Italy

17 INAF Astronomical Observatory of Trieste, Via Tiepolo 11, 34143 Trieste, Italy

${ }^{18}$ Instituto de Astrofisica de Andalucia (CSIC), c/ Glorieta de la Astronomia s/n, 18008 Granada, Spain

${ }^{19}$ Graduate Institute of Astronomy, National Central University, 300 Chung-Da Rd, Chung-Li 32054, Taiwan

${ }^{20}$ Space Science Institute, Macau University of Science and Technology, Avenida Wai Long, Taipa, Macau

${ }^{21}$ Institut für Geophysik und extraterrestrische Physik, Technische Universität Braunschweig, Mendelssohnstr. 3, 38106 Braunschweig, Germany

22 Planetary Science Institute, 1700 East Fort Lowell, Suite 106, Tucson, AZ 85719, USA 